\title{
1 AGL16 regulates genome-wide gene expression and 2 flowering time with partial dependency on SOC1 in 3 Arabidopsis
}

4 Xue Dong ${ }^{1,4}$, Li-Ping Zhang ${ }^{1}$, Dong-Mei Yu ${ }^{1}$, Fang Cheng ${ }^{1}$, Yin-Xin Dong ${ }^{1}$, 5 Xiao-Dong Jiang ${ }^{1,3}$, Fu-Ming Qian ${ }^{1,3}$, Franziska Turck ${ }^{2}$, Jin-Yong $\mathrm{Hu}^{1, *}$

6 1. CAS Key Laboratory for Plant Diversity and Biogeography of East Asia,

7 Kunming Institute of Botany, Chinese Academy of Sciences. Kunming 650201,

8 Yunnan Province, China.

9 2. Department of Plant Development, Max-Planck-Institute for Plant Breeding 10 Research, Carl-von-Linne Weg 10, 50829 Cologne, Germany.

11 3. Kunming College of Life Sciences, University of Chinese Academy of 12 Sciences, Kunming 650201, Yunnan Province, China.

13 4. Germplasm Bank of Wild Species, Kunming Institute of Botany, Chinese

14 Academy of Sciences, Kunming, Yunnan 650201, China

15 * For correspondence: hujinyong@mail.kib.ac.cn. 
18

19

\section{Abstract}

Flowering transition is pivotal and tightly regulated by complex gene-regulatory-networks, in which AGL16 plays important roles. But the molecular function and binding property of AGL16 is not fully explored in vivo. With ChIP-seq and comparative transcriptomics approaches, we characterized the AGL16 targets spectrum and tested its close molecular and genetic interactions with SOC1, the key flowering integrator. AGL16 bound to promoters of more than 2000 genes via CArG-box motifs that were highly similar to that of SOC1. Being consistent with this, AGL16 formed protein complex and shared a common set of targets with SOC1. However, only very few genes showed differential expression in the agl16-1 loss-of-function mutant, whereas in the soc1-2 knockout background, AGL16 repressed and activated the expression of 375 and 182 genes, respectively, with more than a quarter of the DEGs were also bound by AGL16. AGL16 targeted potentially to about seventy flowering time genes involved in multiple pathways. Corroborating with these, AGL16 repressed the flowering time stronger in soc1-2 than in Col-0 background. These data reveals that AGL16 regulates gene expression and flowering time with a partial dependency on SOC1 activity. Moreover, AGL16 participated in the regulation of water loss and seed dormancy. Our study thus defines the AGL16 molecular spectrum and provides insights underlining the molecular coordination of flowering and environmental adaptation. 
41

42

43

44

\section{Introduction}

Timely transitions from vegetative to reproductive growth (floral transition) and from dormant to germinating seeds (dormancy release) determine the capacity of plants to adapt to changing environments, thus these processes are under tight control by complex interactions between endogenous signals and exogenous environmental factors (Andres and Coupland 2012; Michaels 2009; Nee, Xiang, and Soppe 2017). The gene-regulatory-network (GRN) controlling floral transition converges at several floral integrator genes like SUPPRESSOR OF CONSTANS 1 (SOC1) and FLOWERING LOCUS T (FT). These genes often encode transcription regulators controlling the transcription of their downstream targets by binding to specific cis-motifs, for example CArG-boxes (Andres and Coupland 2012; Michaels 2009; Fornara, de Montaigu, and Coupland 2010). CArG-box motifs are binding sites specific for MADS-box transcription factors (TFs) like SOC1, FLOWEING LOCUS C (FLC), SHORT VEGETATIVE PHASE (SVP) and SEPALLATA 3 (SEP3) (Gregis et al. 2013; Mateos et al. 2015; Mateos et al. 2017; Kaufmann et al. 2009; Deng et al. 2011; Immink et al. 2009; Immink et al. 2012; Kaufmann et al. 2010; Tao et al. 2012; Aerts et al. 2018). These MADS-box TFs often form homo- and/or hetero- protein complexes that act in concert and bind to the CArG-box motifs in promoters of more than hundreds of downstream genes to regulate flowering time and other developmental processes of Arabidopsis thaliana.

SOC1 is one key flowering promoter integrating signals from photoperiod, temperature, hormones and age-related pathways (Lee and Lee 2010). SOC1 forms protein complex with AGL24 to activate $L F Y$ and AP1 to initiate and maintain flower meristem identity but represses SEP3 to prevent premature differentiation of floral meristem (Lee et al. 2008). SOC1 can promote the expression of TARGET OF FLC AND SVP1 (TFS1) via recruiting histone demethylase RELATED TO EARLY FLOWERING 6 (REF6) and chromatin remodeler BRAHMA (BRM), and cooperates with SQUAMOSAL PROMOTER BINDING PROTEIN-LIKE 15 (SPL15) to modulate their targets expression thereby regulating flowering time (Richter et al. 2019; Hyun et al. 2016). SOC1 
72 forms a set of heterologous complexes with other MADS-box transcription

73 factors, for example AGL16 (de Folter et al. 2005; Immink et al. 2009).

74 Furthermore, SOC1 times flowering downstream of several hormone signaling 75 pathways including GA, ABA and BRs (Hwang et al. 2019; Jung et al. 2012; Li 76 et al. 2017) and of nutrient status (Yan et al. 2021; Olas et al. 2019; Liu et al. 77 2013). Interestingly, profiling of SOC1 targets also identifies genes involved in 78 the signaling processes of these hormones and nutrients (Immink et al. 2012; 79 Tao et al. 2012). However, the biological significance of these molecular 80 interactions remains to be explored further.

AGL16 represses flowering with dependency on the genetic background, the photoperiod of growth conditions, and gene dosages in A. thaliana (Hu et al. 2014). Only under the inductive long-day conditions loss-of-function mutants for AGL16 show early flowering especially in the functional FRI-FLC background (Johanson et al. 2000; Michaels and Amasino 2001; Hu et al. 2014). AGL16 expression can be modulated by the level of the Brassicaceae-specific miR824, for which natural variation has been reported (Hu et al. 2014; Kutter et al. 2007; de Meaux et al. 2008; Fahlgren et al. 2007; Rajagopalan et al. 2006). Interestingly, changes in miR824 expression result in a significant modification of the plant flowering (Hu et al. 2014). AGL16 acts in flowering time regulation via transcriptional regulation of $F T$, whose expression is also regulated by other MADS-box repressors such as SVP and FLC and other TFs (Aukerman and Sakai 2003; Searle et al. 2006; Jung et al. 2007; Castillejo and Pelaz 2008; Li et al. 2008; Mathieu et al. 2009). AGL16 forms complexes with SVP and FLC, and mildly represses their expression (Hu et al. 2014). AGL16 is a direct downstream target of both FLC and SVP, but the expression of AGL16 changes only weakly in loss-of-function mutants of both genes (Deng et al. 2011; Gregis et al. 2013; Mateos et al. 2015). Yeast-two-hybrids assays suggest that AGL16 interacts with SOC1 and other MADS-box TFs and it has been hypothesized that AGL16 could modulate the

101 SOC1 expression (de Folter et al. 2005; Immink et al. 2012; Immink et al. 102 2009). However, the exact AGL16 target spectrum and the impact of 103 interactions between AGL16 and its partners remain under-explored. 
104 In this study, we examined the molecular profiles that AGL16 bound and tested

105 the molecular and genetic interactions between AGL16 and SOC1. We found

106 that, in contrast to its mild effects in flowering time regulation in Col-0

107 background, AGL16 could in fact bind to more than 2000 target genes that

108 were involved in regulation of flowering time and other biological processes.

109 We confirmed the molecular and genetic interactions of AGL16 with SOC1 and

110 found that they shared many common targets. We demonstrated that the

111 regulatory roles of AGL16 on genome-wide gene expression and flowering

112 time depended partially on the SOC1 activity. 


\section{Results}

\section{AGL16 binds to a large set of genomic segments with CArG boxes}

116 We profiled AGL16 binding sites by a ChIP-seq approach (chromatin

117 immuno-precipitation followed by sequencing). We used a line expressing

118 AGL16 fused to a combined Yellow Fluorescent Protein (YFP) -HA epitope tag

119 under the control of the Cauliflower Mosaic Virus 35S promoter (AGL16OX),

120 which restores the early flowering of agl16-1 to wild type Col-0 level (Fig. S1)

121 (Hu et al. 2014). In two independent trials, we identified respectively 5463 and

1223294 DNA segments statistically enriched for AGL16 binding, of which 3086

123 were shared (Table S2, S3). Most of the peaks were around 150-500 bp in

124 both trials (Fig. S2). To test whether these segments were real binding sites for

125 AGL16, we carried out ChIP-qPCR assays with two independent chromatin

126 preparations for 20 peaks identified by ChIP-seq. These efforts confirmed 12

127 regions bound by AGL16-YFP-HA with a minimum two-fold enrichment in the

128 AGL16OX line compared to agl16-1 background (Fig. 1). Hence, a majority

129 proportion of peaks detected via ChIP-seq method were reproducibly

130 enriched.

131 Peaks bound by AGL16 were annotated using Arabidopsis TAIR10 data to 132 profile their distribution to genomic features (Fig. 2). The peaks from both trials

133 were centered to the $3 \mathrm{~Kb}$ regions around transcriptional start sites (TSS; Fig.

134 2B). Around $60 \%$ of peaks located in the $1 \mathrm{~Kb}$ regions surrounding TSS (Fig.

$1352 \mathrm{C}$; Table S3). About $10 \%$ of peaks were located in the 1-2 kb promoter

136 regions upstream of TSS, while $10-12 \%$ of peaks were in exons/introns. Thus,

137 AGL16 bound to DNA fragments close to TSS of a large set of genes. The

1382339 genes with peaks mapped to gene body or up to $2 \mathrm{~Kb}$ upstream of their

139 TSS were taken as AGL16 targets (Table S3).

140 We next searched for potential cis-elements in the common peaks bound by

141 AGL16 using HOMER, which could predict new motifs and identify known

142 motifs (Heinz et al. 2010). This analysis reported a de novo CArG-box motif 
143 CCATTTTTGG for AGL16 in 707 peaks (24.2\% of all common peaks; Fisher

$144 P=1 \mathrm{e}-340$, in comparison to $3.8 \%$ at genome level; Fig. 2D, Table S3). Ten

145 other CArG-box motifs were also significantly enriched, and matched to the

146 known motifs of SVP, SOC1, SEP3, TAGL1, AGL63, and other MADS-box TFs,

147 most of which could potentially interact with AGL16 (Fig. 2D; Fig. S3; Table S3).

148 The de novo and the ten significantly enriched CArG-box motifs were all

149 distributed around the peak center, indicating that AGL16 bound to its targets

150 via the cluster of CArG-box motifs, just like SOC1 and other MADS-box

151 proteins did (Tao et al. 2012; Immink et al. 2012; Deng et al. 2011). There were

152 also other motifs significantly enriched in the AGL16 bound peaks, such as

153 those bound by TCPs (321 peaks), bHLHs (1131), C2C2 DOFs (2524),

154 WRKYs (1039). However, these motifs were not in the peaks center. Since

155 AGL16 modulated significantly the flowering time in Arabidopsis (Hu et al.

156 2014), we next asked which flowering time genes could be targeted by AGL16.

157 AGL16 targets flowering time genes in multiple pathways

158 The Arabidopsis genome contains $\sim 400$ flowering time genes, among which 159 around 70 were targeted by AGL16 (Fig. 3; Table S3). This number was 160 significantly larger than randomly expected (Yates' Chi-square test, $\mathrm{p}<0.0001$ ).

161 Consistent with the described photoperiod dependency for AGL16-mediated

162 flowering regulation (Hu et al. 2014), 37 genes (for example AGAMOUS LIKE 163 15/16/18 (AGL15/AGL16/AGL18), CONSTANS LIKE 1/3/4/5 (COL1/3/4/5), 164 TWIN SISTER OF FT (TSF) and MOTHER OF FT (MFT), etc.) were related to 165 photoperiod and circadian clock pathways (Bouche et al. 2016). Ten genes 166 (like AGL19 and SVP, etc.) were in the vernalization and ambient temperature 167 pathway, seven genes were involved in the Gibberellin Acid (GA) pathway, and 168 nine genes are integrators or related to meristem response and developmental 169 process. Four genes bound by AGL16 were not clearly defined for the 170 flowering pathways (Zhao et al. 2011; Boxall et al. 2005; Xiao et al. 2009). It 171 should be further noted that, besides GA, jasmonate acid (JA) signaling could 172 also time flowering as well (Kazan and Manners 2013; Zhai et al. 2015; Wang 173 et al. 2017; Bao et al. 2019). Genes in this pathway were directly targeted by 174 AGL16 (Table S3), thus it's possible that AGL16 modulates flowering time also 
175 through this pathway. Taken together, AGL16 might impact several flowering

176 pathways, and the alteration of flowering time in mutants of AGL16 could be a

177 net effect of multiple flowering pathways.

\section{AGL16 binds to SOC1 promoter}

179 The floral integrator gene SOC1 was one of the targets bound by AGL16 (Fig.

180 4A; Table S3). AGL16 interacted with three DNA segments (peaks 1389, 1390

181 and 1391) in the promoter region of SOC1 that harbored several CArG-motifs.

182 Peak 1390 overlapped with a region bound by SOC1 itself (SOC1 binding

183 region 1) (Tao et al. 2012), while peak 1389 overlapped with regions previously

184 shown to be targeted by SVP (Tao et al. 2012; Mateos et al. 2015) or FLC

185 (Deng et al. 2011; Mateos et al. 2015). An independent ChIP-qPCR assay

186 confirmed AGL16 binding on all three peaks with the binding on peaks 1389

187 and 1391 relatively stronger than on peak 1390 (Fig. 5B). The second segment

188 bound by SOC1 itself (SOC1 binding region 2 or fragment 7 ) was not targeted

189 by AGL16. As AGL16 forms protein complexes with SVP and FLC (Hu et al.

190 2014), it is likely that AGL16 binds target regions together with these two

191 MADS-box proteins, thereby participating in modulating target expression.

192 However, the SOC1 transcription was only weakly affected by loss-of-function

193 of AGL16 in the Col-0 background and not significantly in the Col-FRI

194 background (Fig. 5C; Fig. S4), a pattern that had also been observed for SVP

195 (Hu et al. 2014).

196 AGL16 can form protein complex with SOC1 and co-targets a common 197 set of genes

198 Previously, AGL16 was been demonstrated to form heterodimer with SOC1 199 (Fig. S10) (de Folter et al. 2005; Immink et al. 2009). We verified this 200 interaction with Yeast-2-Hybrid (Y2H) and bimolecular fluorescence 201 complementation (BiFC) techniques. $\mathrm{Y} 2 \mathrm{H}$ assays confirmed interactions 202 between SOC1 and AGL16 (Fig. 5A), which was as strong as the previously 203 reported direct interaction between AGL16 and SVP (Hu et al. 2014). LHP1 
204 was used as a negative control. BiFC fusing the $\mathrm{N}$-terminal half of yellow

205 fluorescent protein (nYFP) with AGL16 (35S:AGL16-nYFP) and the C-terminal

206 of YFP with SOC1 (35S:SOC1-cYFP) detected an interaction of AGL16 with

207 SOC1 in the nuclei of Agrobacterium infiltrated tobacco leaves (Fig. 5B).

208 Hence AGL16 and SOC1 can form complexes, which may contribute to the

209 regulation of the expression of downstream targets.

210 We next examined whether AGL16 and SOC1 had common targets. For this

211 aim, the previously generated binding profiles for SOC1 were used to identify

212 shared targets with AGL16 (Immink et al. 2012; Tao et al. 2012). We applied

213 the same annotation procedure for both AGL16 and SOC1 binding profiles in

214 order to identify common genes. There were 193 AGL16 bound segments that

215 overlapped with 240 SOC1 peaks (Table S4). These peaks were in the $+/-2 \mathrm{~Kb}$

216 vicinity of 223 genes (five without annotation information), which were then

217 taken as AGL16 and SOC1 common targets (Fig. 6A). Most of these common

218 peaks were in the $1 \mathrm{~kb}$ region surrounding TSS with AGL16 peaks a bit more 219 proximal (Fig. 6B). We further identified 211 CArG-box motifs in 144 common

220 peaks (400 bp surrounding peak centers; $74.6 \%$ of all overlapped peaks) with

221 MEME-ChIP. Eighty-seven peaks harbored one CArG-box

222 (DCCAAAAAWGGAAAR; 60.4\%), while the rest featured two (49 or $34 \%$ ) or

223 three (6 peaks or $4.2 \%$ ) or more (2 peaks; Fig. S5A). The distances between

224 the CArG-box motifs were significantly spaced with 20-40 bases (Fig. S5B).

225 Among these common targets, genes involved in floral organ development (or

226 reproductive growth) and responses to hormone stimulus including ethylene

227 and ABA were significantly enriched (Fig. 6C; Table S4). Eight genes of the

228 photoperiod and circadian clock related pathways (AGL15, AGL18, ATC,

229 PHYA, RAV2, SMZ, SNZ and TOE3), three genes of the temperature-related

230 pathways (CBF1, CBF2 and SVP), and SOC1 itself were involved in flowering

231 (Fig. 3), indicating that AGL16 and SOC1 could act together to time floral

232 transition in Arabidopsis.

233 The AGL16-SOC1 module is important for genome-wide gene expression 234 and flowering time regulation 
235 As AGL16 and SOC1 formed heteromeric protein complexes and as their

236 genetic interaction played a role in the regulation of AGL16 expression, we

237 determined to what extent the gene expression at the genome-wide level could

238 be affected by the AGL16-SOC1 module (Table S2). For this, we carried out a

239 comparative transcriptomics analysis using the single and double mutants

240 between the agl16-1 and soc1-2 lines. In contrast to the very broad binding

241 spectrum of AGL16, we only detected very small number of genes showing

242 differential expression (DEGs) in agl16-1 single mutant (9 up and 12 down)

243 compared to Col-0 (Fig. 7A; Table S5). The soc1-2 single (155 up and 285

244 down) and the agl16 soc1 double (49 up and 353 down) mutants had similar

245 number of DEGs but soc1-2 featured more up and less down DEGs (Yate's

246 chi-square test, $p<0.001 ;$ Fig. 7A), indicating that AGL16 either countered

247 SOC1's repressive role on gene expression or its inductive role. A heatmap

248 analysis of DEGs in the soc1-2 vs Col-0 revealed that absence of agl16 mostly

249 reverted the differential gene expression observed in soc1-2 to wild type levels

250 (Fig. 7B). Genes down-regulated in the agl16 soc1 mutants showed also

251 down-regulation in soc1-2 (Fig. 7C). In contrast, genes up-regulated in agl16

252 soc1 were barely affected by either single mutation, suggesting that for these

253 genes, AGL16 and SOC1 synergistically contribute to the repression.

254 Accordingly, only 83 soc1-2 DEGs (in total 155 up and 285 down; 18.9\%)

255 overlapped with the agl16 soc1 DEGs (375 up and 182 down; 14.9\%; Fig.

256 7D). Therefore, AGL16 has an important potential in regulating gene

257 expression at the genome-wide level, but apparently depends on its genetic

258 background, here, the SOC1 activity.

259 We next examined to what extent these DEGs associated with AGL16

260 targeting. Among the 557 agl16 soc1 DEGs, AGL16 bound to 98 genes

$261(\sim 22.2 \%)$, of which only $23(\sim 4.1 \%)$ were also targeted by SOC1 (Fig. 7D).

262 About $13.6 \%$ or 60 soc1-2 DEGs were likely the AGL16 targets (Yate's

263 chi-square test, $p=2 e-8$, in comparison to genome-wide level of AGL16

264 binding). However, we noticed that only nine soc1-2 DEGs ( 2\% among 440)

265 were potential targets of SOC1, a pattern similar to a previous report, in which

26652 SOC1 targets were among the 1186 DEGs (Tao et al. 2012). There were six

267 targets $(\sim 28.6 \%)$ showing differential expression in the 21 agl16-1 DEGs. 
268 Moreover, we identified more than a quarter of up-regulated DEGs specifically

269 in the agl16 soc1 (77 among 286) were AGL16 targets in contrast to about

$27013.3 \%$ of up-regulated DEGs specifically in the soc1-2 mutant (29 among 218;

271 Yate's chi-square test, $p=0.0035$; Fig. 7E). Among the 67 up-regulated DEGs

272 shared between the soc1-2 and agl16 soc1 mutants, 18 (26.9\%) were

273 potentially AGL16 targets. However, only less than $8 \%$ of down-regulated

274 DEGs in both mutants were potentially targeted by AGL16. Together, these

275 data suggest that AGL16 may act mainly as a transcriptional repressor in the

276 soc1-2 background.

277 Among the DEGs between agl16 soc1 and soc1-2 plants, we identified 17

278 known genes involved in flowering time regulation with seven of them (NF-YA2,

279 TCP2 1, RHL41, AGL16 and three AP2-like genes RAV1, RAV2/TEM2, and 280 SNZ) being targeted by AGL16 (Fig. 7F; Table S5). Expression of FT was 281 significantly enhanced in agl16 soc1 double mutant. In line with this, the 282 double mutant agl16 soc1 flowered significantly earlier ( 20 rosette leaves) 283 than the soc1-2 single mutant ( $\sim 25.6$ rosette leaves; about $21.6 \%$ reduction in 284 rosette leaf number) but still later than both agl16-1 ( $\sim 11.1$ rosette leaves; $285 \sim 13.6 \%$ reduction) and wild type Col-0 ( 12.9 rosette leaves) plants (Fig. 8).

286 This indicated that AGL16 could counteract SOC1 effects in flowering time 287 regulation. Thus, the regulatory role of AGL16 in floral transition depends on 288 SOC1 function, similar to the genetic dependency of AGL16 on FLC (Hu et al. 289 2014). It's possible that SOC1 repressed the inhibition of AGL16 on FT 290 expression, which should be tested further.

\section{AGL16 is involved in water loss and seed germination regulation}

292 Given the very broad binding profile at the genome-wide, we continued to 293 explore whether AGL16 played a regulatory role in other biological process. 294 AGL16 binds to a large set of genes involved in abscisic acid (ABA) signaling 295 (29), and ABA (101) and water (62) responses (Table S3). Since the function of 296 ABA in regulating adaptation to water availability has been well established, 297 we questioned whether AGL16 could have a role in water governance. We 
298 used the agl16-1 and $m 3$, a line in which the AGL16-specific negative regulator

299 miR824 was highly expressed (Hu et al. 2014; Kutter et al. 2007), to examine

300 the water-loss-rate in the aerial parts of Arabidopsis plants after cutting.

301 Compared to Col-0 control plants, six-weeks-old short-day grown mutant

302 plants displayed a weak but significant decrease in water loss $(2-4 \%$;

303 Student's t-test, $\mathrm{p}<0.001$; Fig. 9A), suggesting that miR824-regulated AGL16

304 could regulate the response to water deficiency. The change in water loss

305 could be either caused by the reduction of stomata density (Kutter et al. 2007)

306 and/or by altering the stomata aperture size, which is tightly associated with

307 the ABA signaling pathway (Zhao et al. 2020).

308 ABA plays essential roles in seed germination and dormancy control (Bewley 309 1997; Bentsink and Koornneef 2008). Not surprisingly, a further examination 310 on seed dormancy levels demonstrated a significant alteration in germination 311 rate of freshly harvested seeds of agl16-1 and m3 compared to the wild type, 312 and to a reduced extent, after one-week storage (Fig. 9B). This pattern was 313 tightly associated with increased levels of miR824 and a decreased expression 314 of AGL16 in germinating seeds (Das et al. 2018). Taken together, these data 315 suggest a regulatory role of AGL16 in water adaptation and seed germination. 316 Corroborating with this, AGL16 was recently identified as a negative factor of 317 drought resistance via regulation on stomata density and ABA accumulation 318 (Zhao et al. 2020). CYP707A3 (Zhao et al. 2020), CYP707A1 and AAO2, 319 which are involved in ABA biosynthesis and metabolism, were among the 320 AGL16 targets (Table S3). Since ABA related signaling genes were also 321 enriched in AGL16-SOC1 common targets (Fig. 6C), it would be worth to 322 examine further the regulatory function of the AGL16-SOC1 module in water 323 loss and seed dormancy processes. 


\section{Discussion}

326 In this study, via ChIP-seq and transcriptomic profiling as well as genetic

327 analyses, we show that AGL16 targets to a broad range of genes and acts in a

328 wide range of biological processes such as water deprivation and seed

329 germination time. Depending on SOC1 function, AGL16 occupies important

330 hubs in the GRNs involved in flowering time regulation.

\section{AGL16 interacts with SOC1 and times flowering with a partial}

\section{background dependency on SOC1}

333 AGL16 is known as a floral repressor in photoperiod pathway of flowering time

334 regulation (Hu et al. 2014). Corroborating with previous notions (de Folter et al.

335 2005; Immink et al. 2012; Immink et al. 2009), AGL16 forms heteromeric

336 protein complexes with $\mathrm{SOC} 1$, as evidenced by our $\mathrm{Y} 2 \mathrm{H}$ and $\mathrm{BiFC}$ analyses

337 (Fig. 5). This suggests that both proteins work together to target a common set

338 of downstream genes. We provide evidences that AGL16 binds potentially

339 more than 2000 target genes (Fig. 2), many of which are share with SOC1

$340(\sim 50 \%$ of SOC1 bound genes; Fig. 6). Since AGL16 forms also protein

341 complexes with SVP and FLC (Hu et al. 2014) and potentially with SEP3 (Fig.

342 S3) (de Folter et al. 2005), it would be worth to examine whether AGL16

343 shares also common targets with these TFs. As AGL16 times flowering time

344 with a genetic background dependency on SOC1, similar to our previous

345 finding on AGL16's dependency on FLC activity (Hu et al. 2014), whether

346 SOC1 and FLC work together to mediate the AGL16's function in flowering

347 time regulation awaits further investigation.

348 AGL16 might exert its regulation potential in several pathways controlling

349 flowering time (Fig. 3). Being congruent with its photoperiod dependency in

350 regulation of flowering time, AGL16 targets 37 genes (including AGL16 itself)

351 related to photoperiod and circadian clock pathways. Under the tested

352 environmental conditions agl16-1 still shows a normal vernalization response

353 (Hu et al. 2014), several genes related to temperature responses are directly 
354 targeted by AGL16. FLC, SVP and SOC1 might be partners of AGL16 in this

355 respect as all three proteins target also directly on some of these

356 temperature-related genes (Deng et al. 2011; Mateos et al. 2015; Immink et al.

357 2012; Tao et al. 2012). The binding of AGL16 may cause both positive and 358 negative influences on the transcription of these targets (Fig. 7), which

359 encompass both repressors and promoters of the floral transition. Indeed, 360 several of the flowering time genes targeted by AGL16 show an enhanced or 361 decreased expression when AGL16 activity is modified in the soc1-2 362 background (Fig. 3 and 7; Table S4, S5). Therefore, the early flowering 363 phenotypes present in AGL16 loss-of-function mutants (Fig. 8) (Hu et al. 2014)

364 might be a net-effect/balance of the regulation on different pathways.

\section{AGL16-SOC1 module acts in regulating genome-wide gene expression}

366 MADS-box TFs often act together to target and regulate the expression of a 367 broad set of downstream genes (de Folter et al. 2005; Deng et al. 2011; 368 Immink et al. 2009; Immink et al. 2012; Kaufmann et al. 2009; Kaufmann et al. 2010; Lee et al. 2008; Mateos et al. 2015; Tao et al. 2012). Although AGL16 binds more than 2000 genes, which is in line with its very broad expression in many tissues and organs (Alvarez-Buylla et al. 2000), AGL16 alone can only

372 affect the expression of a limited number of genes in the background of Col-0, 373 in which SOC1 is functional (Fig. 7). However, when SOC1 is non-functional 374 (in soc1-2 background), AGL16 modulates the expression of more than 550 375 genes and acts both as a transcriptional repressor and activator. Moreover, in 376 the soc1-2 background, AGL16 seems mainly act as a transcriptional 377 repressor as more than a quarter of the up-regulated DEGs, in contrast to the 378 less than $8.5 \%$ of the down-regulated DEGs, are potential targets of AGL16. 379 Hence AGL16's activity in gene expression regulation requires partially the 380 participation of SOC1. On the other hand, SOC1 also needs partially the 381 AGL16 function as SOC1's repressive activity significantly drops (from 155 to 38249 genes) but the promoting activity significantly increases (from 285 to 353 383 genes) when AGL16 has no function. Many soc1-2 DEGs are not differentially 384 expressed any more in agl16 soc1 mutant (Fig. 7). Therefore, AGL16 and 385 SOC1 act both additively and synergistically in regulation of genome-wide 
386 gene expression.

387 AGL16 is important in GRNs connecting life-history traits

388 Both AGL16 and SOC1 can directly bind to chromatin and regulate the 389 expression of genes involved in hormone signaling and abiotic stresses (Fig. 6) 390 (Immink et al. 2012; Tao et al. 2012). Corroborating with this, alteration in 391 AGL16 activity significantly changes the water loss efficiency, a process for 392 which stomata development (Kutter et al. 2007) and ABA signaling might play 393 a role (Zhao et al. 2020). Previously, SOC1 has been implicated in modulating 394 stomata opening (Kimura et al. 2015). AGL16 also participates in the 395 regulation of seed germination (Fig. 9), a key step in plant life cycle and 396 adaptation to fluctuating environmental conditions (Koornneef, Bentsink, and 397 Hilhorst 2002; Bewley 1997). The expression of miR824-regulated AGL16 398 decreases significantly during seed germination (Das et al. 2018). The 399 regulatory role of AGL16 in seed germination might be related to ABA as many 400 ABA signaling genes including those encoding for ABA receptors, such as 401 PYL4, PYL5, PYL8, are directly targeted by AGL16 (Table S3). PYL8 is 402 co-bound by SOC1 (Table S4). Therefore, the miR824-AGL16 module seems 403 to be important in GRNs connecting the two key transitional events, i.e., 404 flowering and germination.

405 In summary, our data reveals that, as a master regulator in GRNs connecting 406 multiple biological pathways, AGL16's function depends partially on SOC1, 407 similar to the genetic dependency on FLC (Hu et al. 2014). AGL16 might act as 408 a glue, like other MADS-box TFs do, to modulate the chromatin accessibility of 409 their interacting proteins to micro-tune the expression of downstream genes at 410 proper stages and environmental conditions (Pajoro et al. 2014; Immink et al. 411 2009; Kaufmann et al. 2010; Richter et al. 2019). It will be important to address 412 this further to understand their precise roles and mechanisms in balancing 413 development and adaptation.

\section{Materials and methods}


415 Plant materials, and growth conditions

416 A. thaliana plants including wild-type Col-0, agl16-1, 35S:AGL16-YFP-HA in 417 agl16-1 background, Col-FRI, agl16-1 Col-FRI, and $m 3$ have been described 418 previously (Kutter et al. 2007; Hu et al. 2014). The soc1-2 mutant in Col-0 419 background (Torti et al. 2012) was kindly provided by Prof. George Coupland. 420 To test the genetic interactions between AGL16 and SOC1, agl16-1 and 421 soc1-2 were crossed and double mutant was screened with gene-specific 422 primers (Table S1) (Torti et al. 2012; Kutter et al. 2007; Hu et al. 2014).

423 Arabidopsis seeds were stratified in distilled water at $4^{\circ} \mathrm{C}$ for $72 \mathrm{~h}$ and sown in 424 soil and grown under LD conditions (16-h light at $21^{\circ} \mathrm{C}$ and 8 -h night at $18^{\circ} \mathrm{C}$ ). 425 Seedlings for phenotyping were planted either in growth rooms or chambers, 426 while materials for gene expression analysis and ChIP assays were sown on 427 Murashige and Skoog medium plates (Hu et al. 2014).

428 RNA Isolation, real-Time RT-qPCR, and RNA-seq assays

429 Total RNA was extracted with TRI Reagent ${ }^{\circledR}$ (Molecular Research Center, Inc. 430 Cincinati, USA). Ten days old seedlings were used for quantification of relative 431 expression of selected genes with PP2A as reference (Hu et al. 2014). 432 Reverse transcription was carried out with the HiScript ${ }^{\circledR}$ II Q RT SuperMix for 433 qPCR (+gDNA wiper) and quantification PCRs were performed with ChamQ ${ }^{T M}$ 434 SYBR qPCR Master Mix (both from Vazyme Biotech co. Itd, Nanjing) on 435 QuantStudio ${ }^{\mathrm{TM}} 7$ Flex Real-Time PCR System (ThermoFisher). Three to four 436 biological replicates from each of two to three independent trials were applied 437 for each experiment. A similar protocol was developed for monitoring relative 438 enrichment of DNA fragments in ChIP-qPCR experiments. All the primers used 439 in this study are included in Table S1.

440 For RNA-seq, materials were collected from three independent biological 441 replicates for each genotype, and DNA-free total RNA was generated as 442 described above. Illumina True-seq library preparation was performed from 3 
$443 \mu \mathrm{g}$ DNA-free total RNA and sequenced by the Biomarker Technologies 444 Corporation, Beijing, China. Quality trimmed pair-end RNA-seq reads were 445 mapped to the Arabidopsis TAIR10 annotation using the HISAT2 v2.1.0 (Kim 446 et al. 2019). The featureCounts included in subread v1.6.4 package was 447 applied to calculate reads counts on each gene (Liao, Smyth, and Shi 2013; 448 Liao, Smyth, and Shi 2014). DESeq2 v1.14.1 was used to detect differentially 449 expressed genes (DEGs; fold change above 1.5 and p.adj<0.1). Only uniquely 450 mapped reads were used for downstream analysis. Transcriptional clustering 451 analysis was performed using the heatmap.2 function in $R$. GO analysis was 452 performed with PANTHER in TAIR web-tool 453 (https://www.arabidopsis.org/tools/go term enrichment.jsp) (Mi et al. 2017) or 454 agriGO pipeline (Tian et al. 2017).

\section{ChIP-seq and ChIP-qPCR assays and data analysis}

456 ChIP experiments were carried out following protocols described (Zhou et al. 457 2016; Reimer and Turck 2010). Chromatin for both ag/16-1 and agl16-1 458 AGL16OX plants was extracted from ten-day-old seedlings grown under LD 459 conditions at ZT14, and precipitated with antibody against GFP (Abcam, 460 Ab290). For ChIP-seq, the immuno-precipitations from two independent trials were used for NGS library preparation with NEBNext ${ }^{\circledR}$ Ultra $^{\mathrm{TM}}$ II DNA Library

462 Prep Kit for Illumina ${ }^{\circledR}$ (E7645, New England BioLabs Inc.) and high-throughput 463 sequencing with HiSeq2000 platform. ChIP-seq reads were mapped to the 464 TAIR10 assembly of $A$. thaliana using BWA-MEM (v0.7.17-r1188) (Li 2013). 465 Reads with mapping quality below 30 were discarded with SAMtools v1.7 (Li et 466 al. 2009). Duplicated reads were removed using Picard MarkDuplicates v1.119. 467 The resulted .bam file was used as input to call AGL16 enriched regions with 468 MACS v2.2.7.1 (Zhang et al. 2008). Enriched regions were generated by the 469 comparison of immune-precipitated products to input for AGL16OX and then 470 compared against agl16-1. For annotation of AGL16 targets, the $R$ package 471 ChIPseeker was used (Yu, Wang, and He 2015). The position and strand 472 information of nearest genes were reported with the distance from peak to the 
473 TSS of its closest gene identified. As annotations might overlap, we use

474 'promoter' definition in ChIPseeker as the highest priority for annotation. Each 475 binding site was assigned to only one gene. IGV was used for data 476 visualization of the binding profiles for targets (Thorvaldsdottir, Robinson, and 477 Mesirov 2013). Enriched motifs in AGL16 binding peaks were identified using 478 Homer suite with findMotifsGenome.pl function (Heinz et al. 2010). Motifs in 479 AGL16-SOC1 co-targeted regions were analyzed with MEME-ChIP tools 480 (Machanick and Bailey 2011), and the spacing between primary and 481 secondary motifs was analyzed with SpaMo (spamo -dumpseqs -bin 20 482 -verbosity 1 -oc spamo_out_1 -bgfile./background -keepprimary -primary 483 DCCAAAAAWGGAAAR). We compared the AGL16 targets to SOC1 targets 484 from both Immink (2012) and Tao (2012) with the same annotation procedures 485 for AGL16 (Immink et al. 2012; Tao et al. 2012). In an earlier independent trial, 486 we pooled the immune-precipitations from two biological replicates and 487 sequenced the products. This pooled sequencing results gave similar pattern 488 of AGL16 targets profile but with a lower coverage hence the data was not 489 shown. Yate's chi-square tests were performed online 490 (http://www.quantpsy.org/chisq/chisq.htm). The $\sim 400$ flowering time genes 491 were downloaded from https://www.mpipz.mpg.de (Bouche et al. 2016) with 492 self-curations. Reads data for RNA-seq and ChIP-seq experiments were 493 accessible at NCBI under accession code SUB5067038.

\section{Phenotype assays}

495 Flowering time assays were carried out according to previous report (Hu et al. 496 2014). Four independent trials were applied and each gave similar pattern. 497 Phenotype comparisons were performed with Student's t-test with 498 Bonferroni-correction.

499 For water-loss assays, rosette leaves of six week-old plants grown under short 500 day conditions were used for measurements of water-loss rates (Lefebvre et al. 501 2006). Fresh rosettes were cut at their base and immediately weighted to 502 establish initial fresh weight $\left(\mathrm{FW}_{\mathrm{i}}\right)$. These rosettes were left in open air at room 
503 temperature in the lab and weighted 1, 2, 3, and $4 \mathrm{hrs}$ after cut to calculate

504 weight loss per unit of time, $\left(\mathrm{FW}_{\mathrm{t}}-\mathrm{FW}_{\mathrm{i}}\right)$. At each time point, the amount of water

505 lost was quantified by expressing the lost weight per unit of time as a

506 percentage of $\mathrm{FW}_{\mathrm{i}}$. To quantify the role of the miR824/AGL16 regulatory

507 system in the rate of water loss, average water loss of the mutants was

508 expressed as percentage of the average water loss measured in Col-0. This

509 experiment was repeated two times and both gave similar pattern.

510 For seed dormancy assays, about 50 individually- and freshly- harvested

511 seeds were plated onto a filter paper moistened with demineralized water in

512 Petri dishes and incubated in LD conditions in transparent moisturized

513 containers (16h light $/ 8 \mathrm{~h}$ dark, $25^{\circ} \mathrm{C} / 20^{\circ} \mathrm{C}$ cycle) (Xiang et al. 2014).

514 Germination was scored after 7 days of incubation. For each assay, at least

515 three trials, each with minimum 10 individual plants, were used.

516 Yeast two-hybrid and biomolecular fluorescence complementation (BiFC) 517 experiments

518 Yeast two-hybrid and the BiFC assays were carried out to test the physical 519 interaction between AGL16 and SOC1 proteins according to previous report 520 (Hu et al. 2014). In yeast two-hybrid assay, interactions between AGL16-SVP 521 and AGL16-AGL16 were applied as positive controls while the AGL16-LHP1, 522 AGL16-BD, SOC1-BD, AD-AGL16, and AD-SOC1 were applied as negative 523 controls together with empty vectors. For BiFC assay in Nicotiana 524 benthamiana plants, 35S:SOC1-cYFP construct was built by cloning the

525 full-length encoding-region without stop codon of SOC1 (from Col-0) into 526 pDONR221 entry vector first and later transferred into RfA-sYFPc-pBatTL-B 527 vector. The interactions between AGL16 and SVP, between AGL16 and LHP1, 528 were used as positive and negative controls, respectively.

\section{Acknowledgements}

530 We thank Liangyu Liu, Feihong Yan, Fei He, Yibo Sun, Shulan Chen for 
531 assistance in experiments. This work was supported by grants from National

532 Natural Science Foundation of China (31570311 to J-Y H, 31501034 to X D,

53331700275 to $Y-X$ D, 31800261 to $F$ C), from the CAS Pioneer Hundred Talents

534 Program (292015312D11035 to J-Y H), and CAS Key Laboratory for Plant

535 Diversity and Biogeography of East Asia to J-Y H, from the China Postdoctoral

536 Science Foundation (2017M613023 to Y-X D), the Postdoctoral targeted

537 funding from Yunnan Province to $F \mathrm{C}$ and $\mathrm{Y}-\mathrm{Y} \mathrm{D}$, and the Yunnan basic and

538 applied research funding to $\mathrm{F} C$. This work is partially facilitated by the

539 Germplasm Bank of Wild Species of China. The authors declare no conflict of 540 interest.

\section{Author Contributions}

542 J-Y H conceptualized and coordinated the research; L-P Z performed the ChIP

543 experiments and collected the RNA samples, D-M $Y$ and $Y Z$ carried out the 544 protein interaction assays, $X \mathrm{D}, \mathrm{J}-\mathrm{Y} \mathrm{H}$ and L-P Z created the genetic materials 545 and did the genetic analyses, $X \mathrm{D}$ analyzed and visualized the data, F C, Y-X D,

546 X-D J, F-M Q and F T did other analyses; J-Y H wrote the paper with help from

547 F T and the other authors. All authors had read and approved the manuscript. 
A

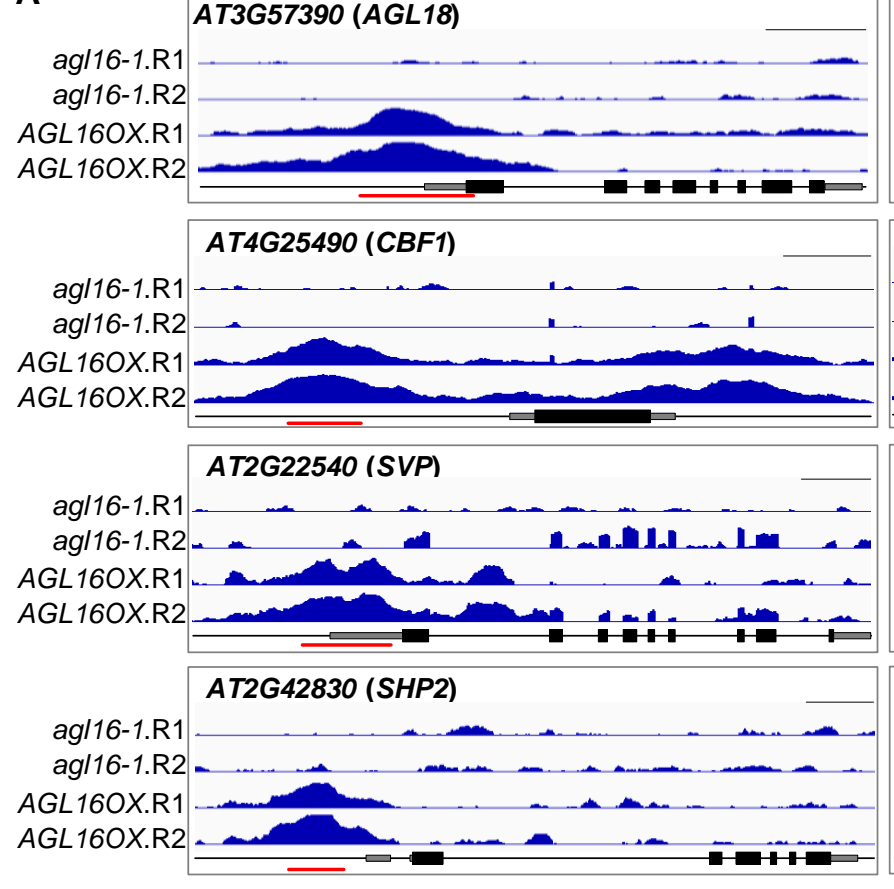

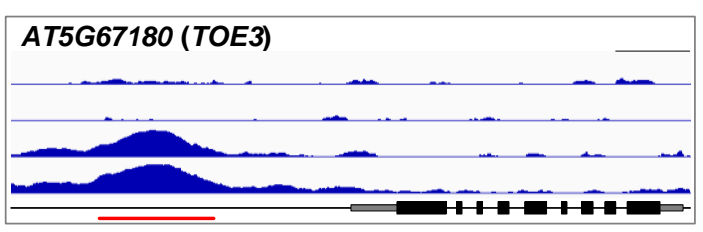
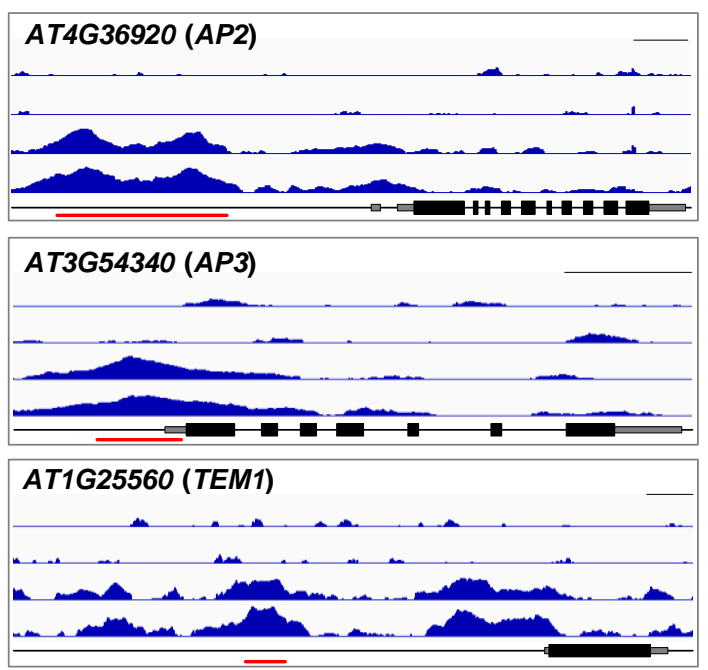

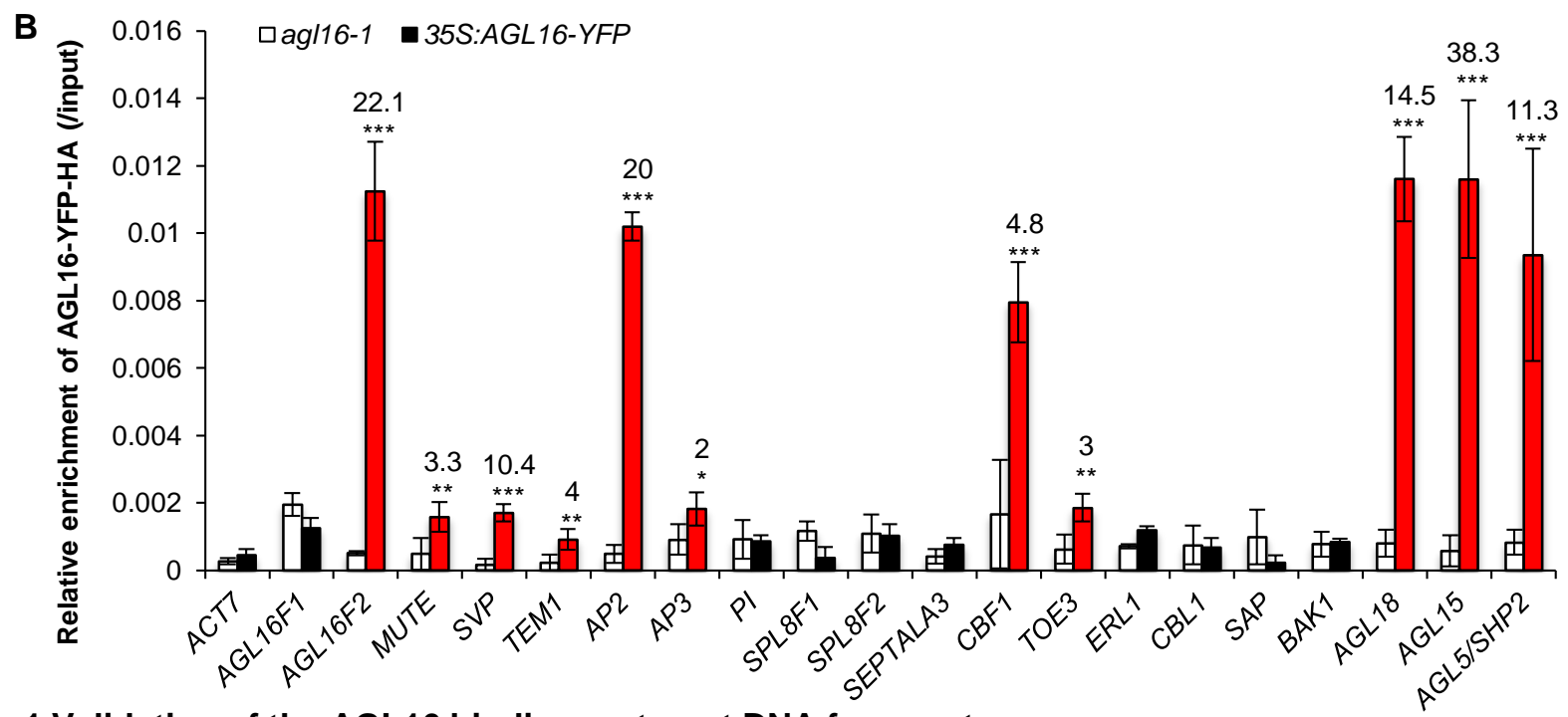

Fig. 1 Validation of the AGL16 binding on target DNA fragments.

A. Binding profiles for selected target genes. The TAIR10 annotation of the genomic locus was shown at the bottom of each box. For each panel, the profiles for two trials (R1 and R2) in agl16-1 background line were shown in the upper panel, while the profiles for agl16-1 35S:AGL16-YFP-HA (AGL16OX; two trials) were shown in the middle panel of each box. All the genes were from 5 '-end to 3 '-end with scale bars indicating sequence lengths of $500 \mathrm{bp}$. Note that data range for each gene in agl16-1 and AGL16OX was the same scale, but different genes could have different scale. Red lines marked the binding regions tested via ChIP-qPCR assays (B).

B. ChIP-qPCR validation of AGL16 binding on 20 DNA segments. Significant enrichment (red bars) was defined with the following criteria: mean enrichment must be at least two-fold higher than negative control $A C T 7$, the enrichment for AGL16OX (in ag/16-1 background) than ag/16-1 must be higher than two-fold change, and the amplification $\mathrm{C}_{\mathrm{T}}$ number of IP samples must be at least 2 cycles less than no-antibody controls. This experiment was repeated with another independent trial, in which the relative enrichment of AGL16F1 and SAP did not meet above criteria (see Figure S4). Statistics was carried out with Student's $t$-test with Bonferroni correction. ${ }^{* * *}$, $\mathrm{P}<0.001 ;{ }^{* *}, \mathrm{p}<0.01 ;{ }^{*}, \mathrm{P}<0.05$. 
A

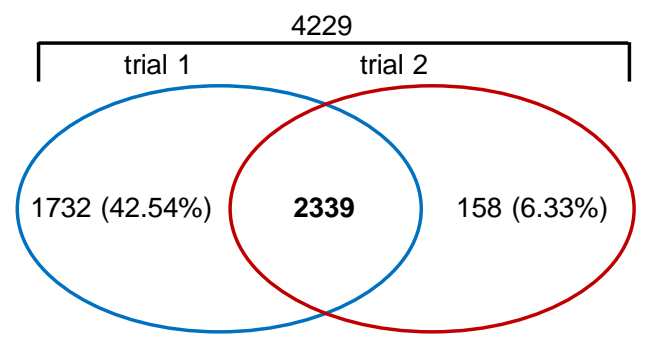

B
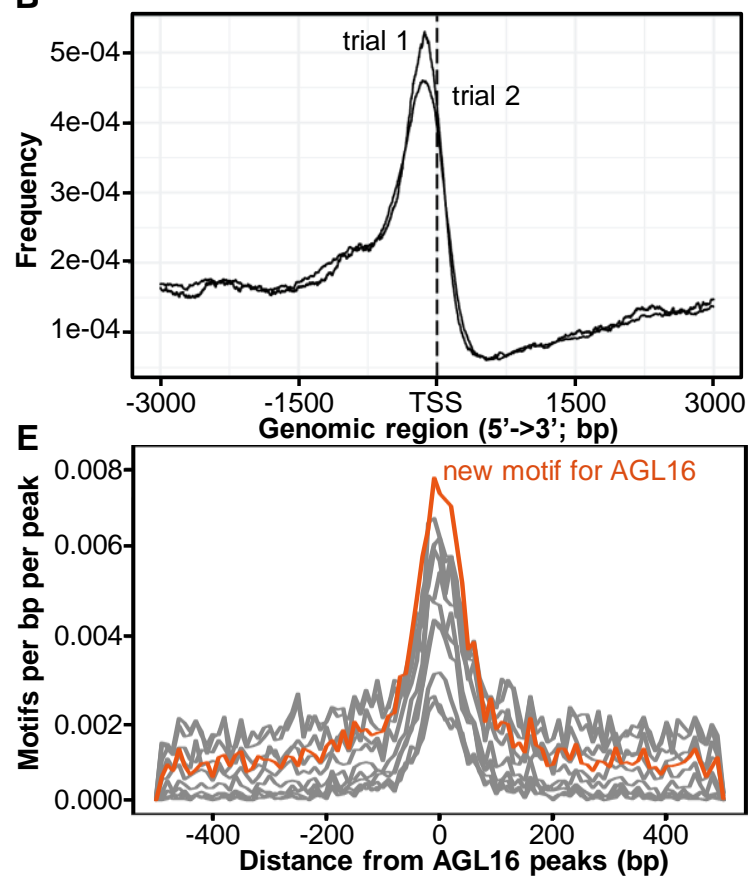

C

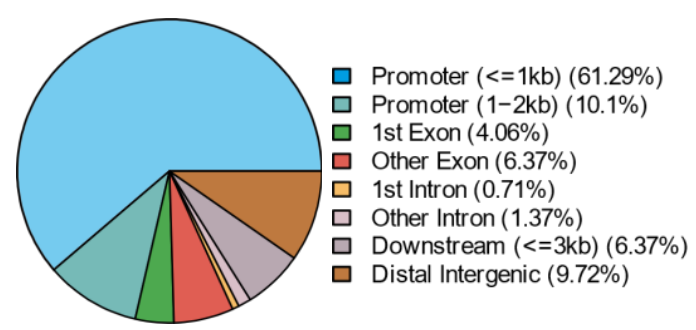

D

\begin{tabular}{|c|c|c|}
\hline Type & Consensus sequence & Frequency \\
\hline AGL16 new & IXCCATIATAGG & $24.19 \%$ \\
\hline SVP & 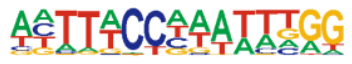 & $19.12 \%$ \\
\hline AGL15 & 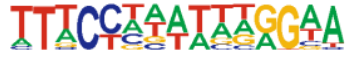 & $10.30 \%$ \\
\hline SOC1 & TACCATATATCG & $14.68 \%$ \\
\hline AGL6 & 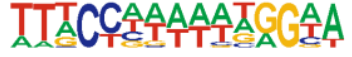 & $10.06 \%$ \\
\hline AGL16 & I즘두송소 & $6.88 \%$ \\
\hline TAGL1 & CСAAAAATGG & $21.35 \%$ \\
\hline AGL25 & IIICCATAAATGGAA & $6.16 \%$ \\
\hline AGL63 & TTCCAAAATTGG & $18.27 \%$ \\
\hline AGL13 & TACCATATATAGGAA & $5.82 \%$ \\
\hline RIN & C두수수수수두도소 & $19.02 \%$ \\
\hline SEP3 & 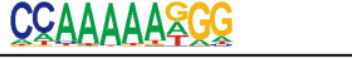 & $13.86 \%$ \\
\hline Proportic & aks with CArG-box motifs & $81.95 \%$ \\
\hline
\end{tabular}

Fig. 2 Genome-wide identification of AGL16 target genes via ChIP-seq.

A. Venn diagram of AGL16 targets identified in two independent trials.

B. Distribution of AGL16 binding sites for two trials surrounding the transcriptional starting site (TSS).

C. Location distribution in relative to nearby genes for AGL16 binding sites of trial 1 . Peaks within the $3 \mathrm{~Kb}$ promoter region were taken as AGL16 targets.

D. CArG type of motifs over-represented in the AGL16 binding peaks. AGL16 new, which was highly similar to known SOC1 type, showed the de novo motif predicted for AGL16. Frequency gave the percentage for each motif presented in the binding peaks.

E. Distribution of new (orange) and known (gray; shown in D) CArG type of motifs around AGL16 peaks center. 


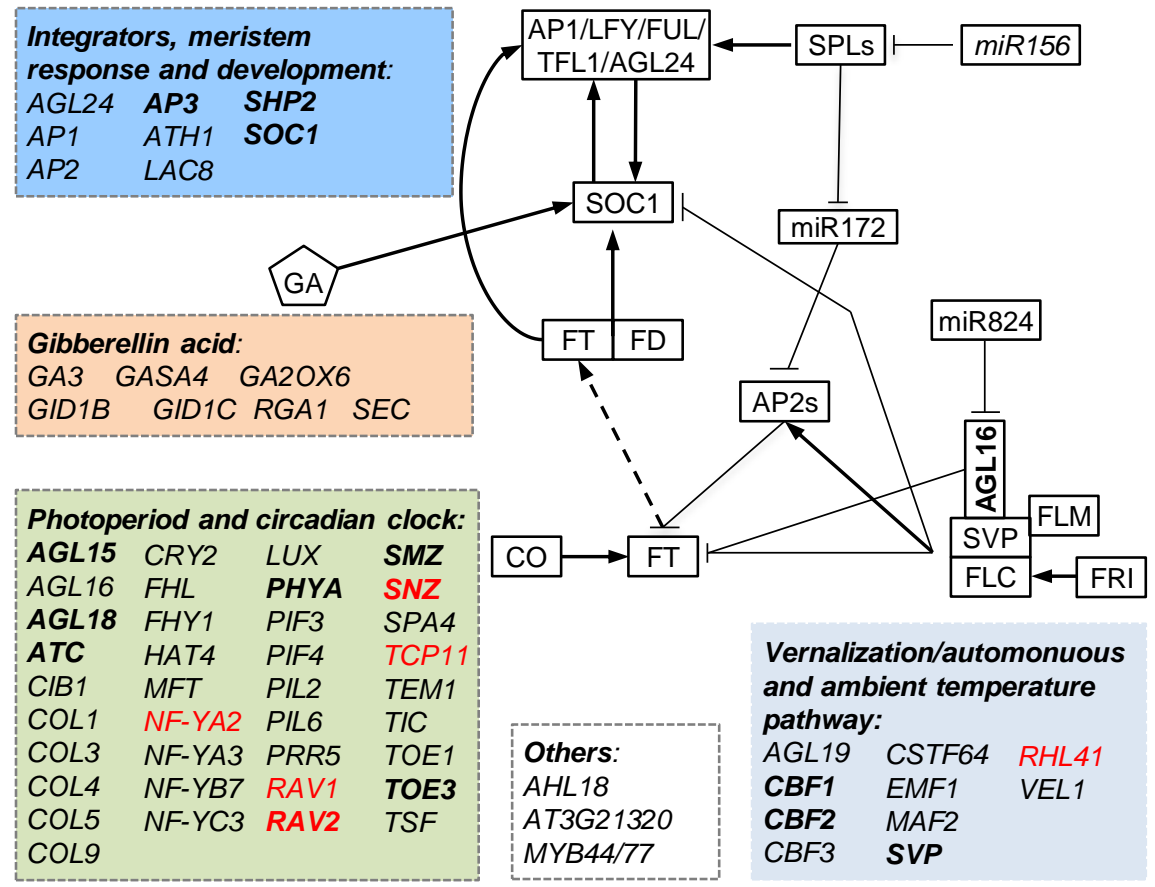

Fig. 3 Molecular pathways (indicated with different color boxes) targeted by AGL16. Genes with names in bold were common targets for AGL16 and SOC1, while those in red were differentially expressed between the ag/16 soc1 and soc1-2 mutants. 
A

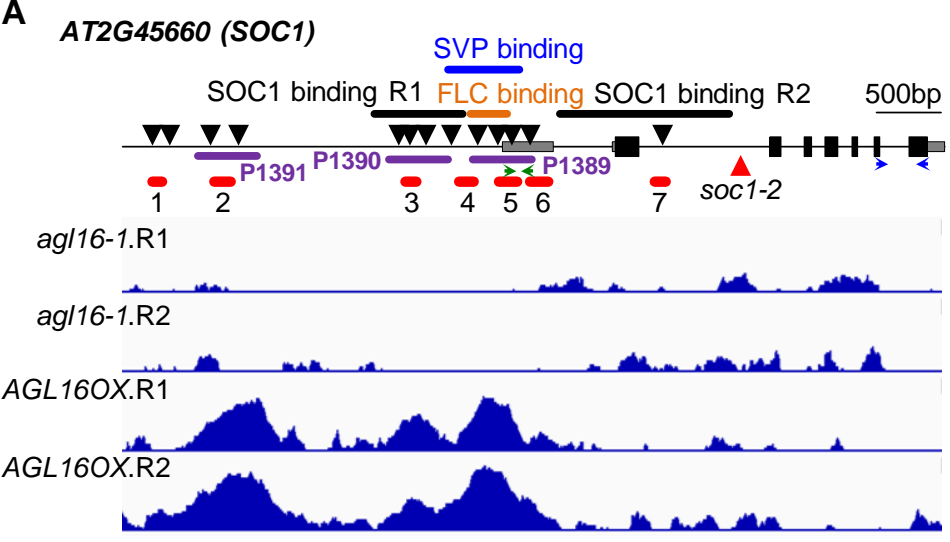

$B$ เ

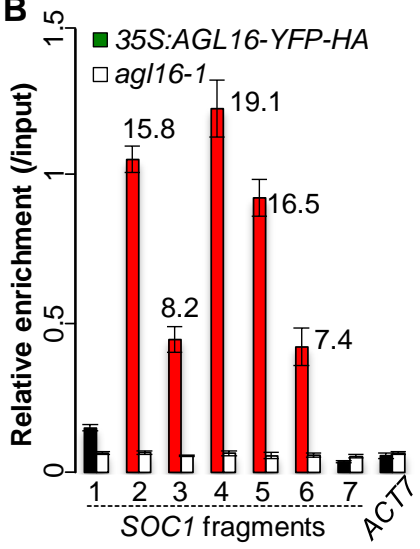

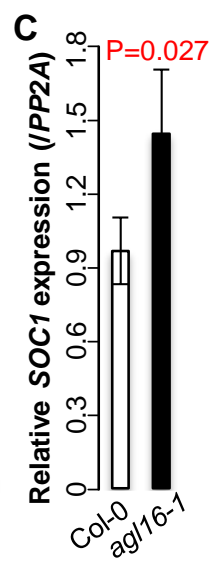

Fig. 4 AGL16 targets and regulates SOC1.

A. Schematic representation of the SOC1 locus. Filled bars indicated exons and gray bars marked the 5and 3-UTR regions while the line indicated the non-coding region of SOC1. Arrows downward labelled the putative CArG-boxes potentially bound by MADS-box proteins. The dark purple lines indicated the three peaks (P1389, P1390 and P1391) bound by AGL16. Orange, blue and black thick lines marked the known regions targeted by FLC, SVP and SOC1, respectively. Note that two sites in the regulatory region of SOC1 were bound by itself (SOC1 binding R1 and R2; see ref. Tao et al. 2012). Red lines (1 to 7) showed the regions tested for AGL16-YFP-HA binding on SOC1 chromatin. Horizontal arrows marked the position of primers used for quantification of 5'-UTR (green) and CDS (blue) regions. The lower panel showed the ChIP-seq profile at SOC1.

B. Relative enrichment of AGL16 on SOC1 chromatin tested with ChIP-qPCR. Fold change values with signficant enrichment was labelled above bars. $A C T 7$ was taken as a negative enrichment control.

C. Relative expression of SOC1 CDS against PP2A in Col-0 and agl16-1 plants. 
A

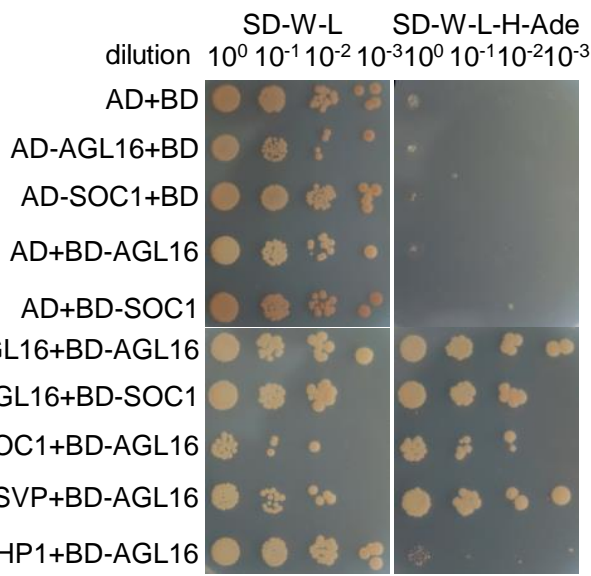

B

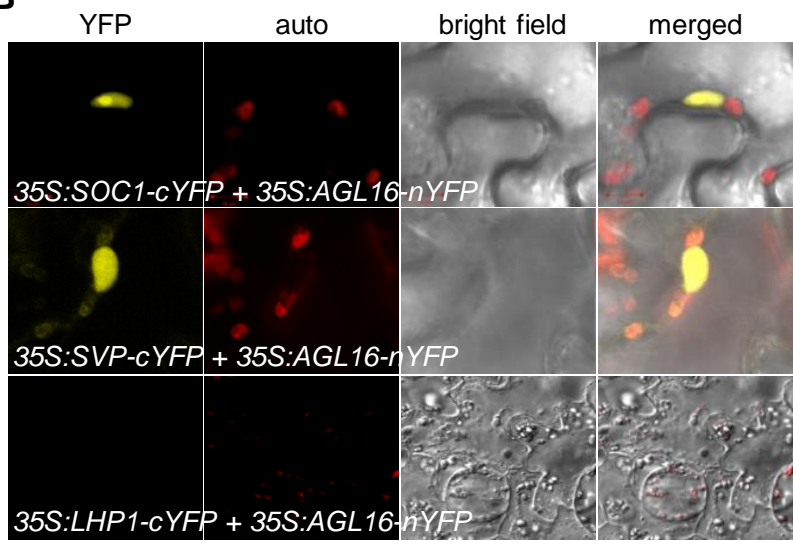

Fig. 5 AGL16 forms protein complex with SOC1.

A. Yeast two-hybrid assay revealed a direct interaction between AGL16 and SOC1. Each protein was fused to either the activation domain (AD) as prey or the DNA-binding domain (BD) as bait. Serial dilutions $\left(10^{0} \times\right.$ to $10^{-3} \mathrm{x}$ ) of $\mathrm{J} 69-4 \mathrm{~A}$ cells containing different construct combinations indicated on the left were grown on control (left) and selective (right) medium. The AGL16-SVP and the AGL16-LHP1/empty vector combinations provided positive and negative controls, respectively. Note the formation of a AGL16 homodimer.

B. BiFC assay evidenced the formation of AGL16-SOC1 complex in nucleus of Nicotiana benthamiana leaf epidermis. The interaction was tested with constructs 35S:SOC1-cYFP and 35S:AGL16-nYFP. A negative interaction between AGL16 and LHP1 and a positive interaction between AGL16 and SVP were tested as well (see also Hu et al. 2014). 

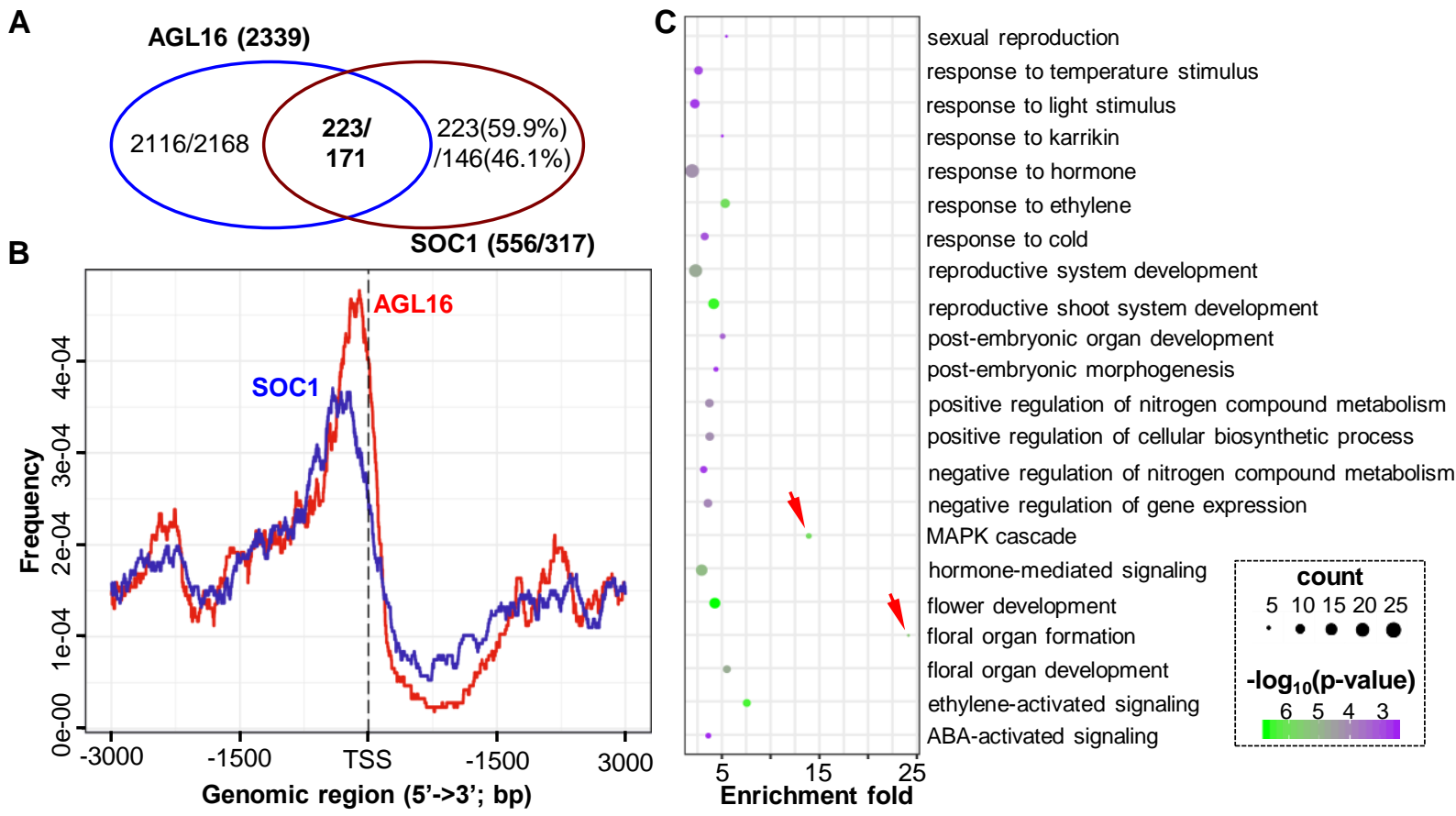

Fig. 6 AGL16 and SOC1 share a common set of target genes involved in multiple functions.

A. Venn diagram showing that 223/171 genes (Immink et al. 2012 / Tao et al. 2012) were co-bound potentially by both AGL16 and SOC1.

B. Binding intensities for AGL16 (red) and SOC1 (blue) peaks surrounding transcription starting sites (TSS). Regions $3 \mathrm{~kb}$ upstream and downstream of TSS were plotted.

C. Selected significantly-enriched GO terms for the common targets. Note the GO terms marked by red arrowheads. 


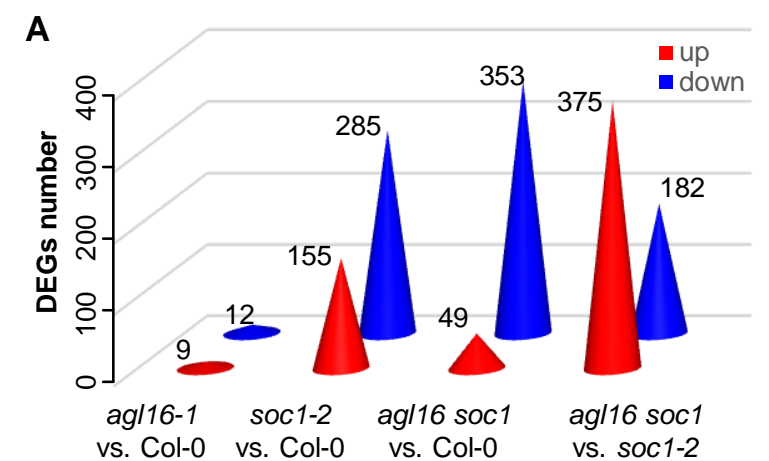

D

B

soc1-2 vs. Col-0 up (285)

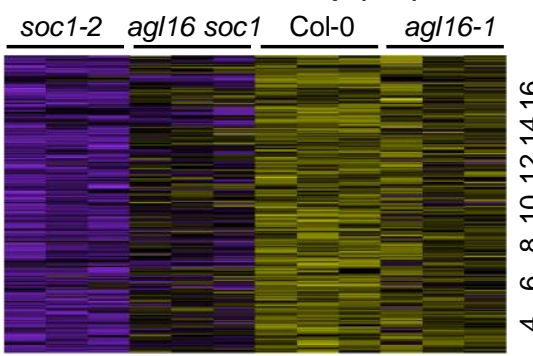

$+2,160^{0^{c}}$

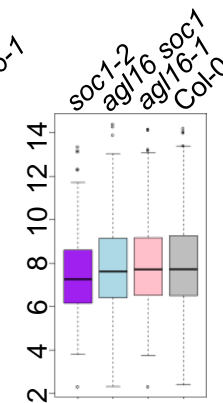

agl16 soc1 DEGs

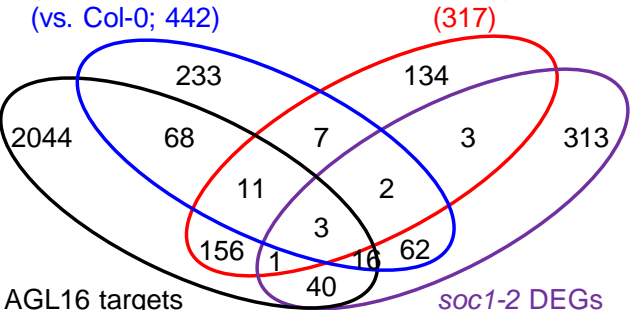

(2339)

(vs. Col-0; 440)

soc1-2 vs. Col-0 down (155)

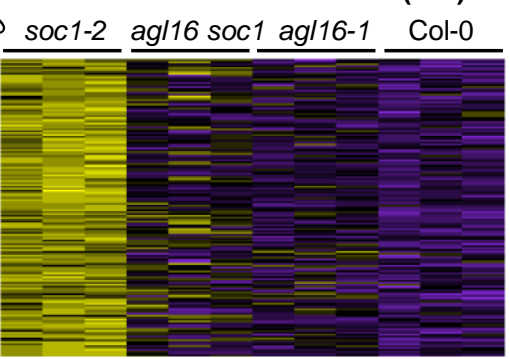

C

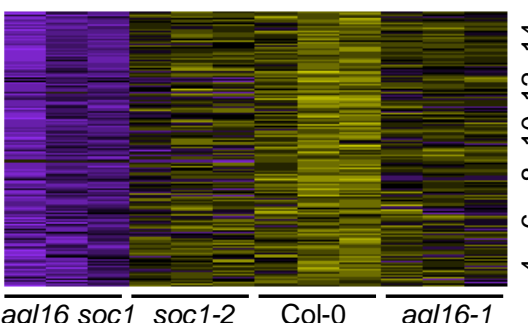

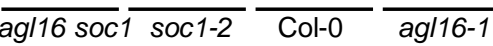

agl16 soc1 vs. Col-0 up (353)

E
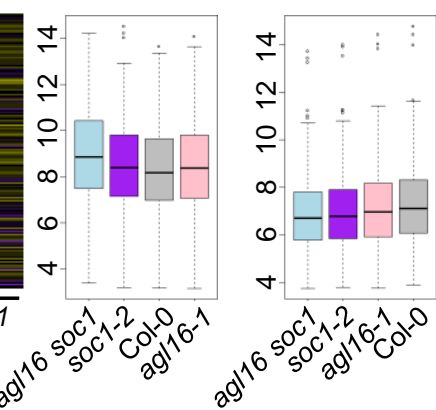

E

agl16 soc1 down

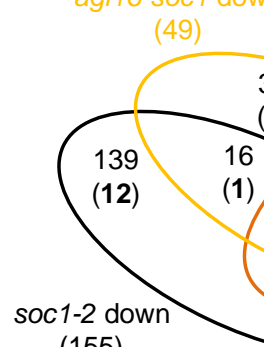

(155) agl16 soc1 up (353)

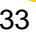

(2)

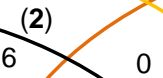

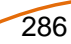

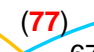

(18)

218

(29)

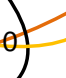

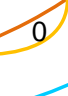

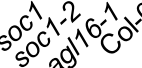

$\mathbf{F}$

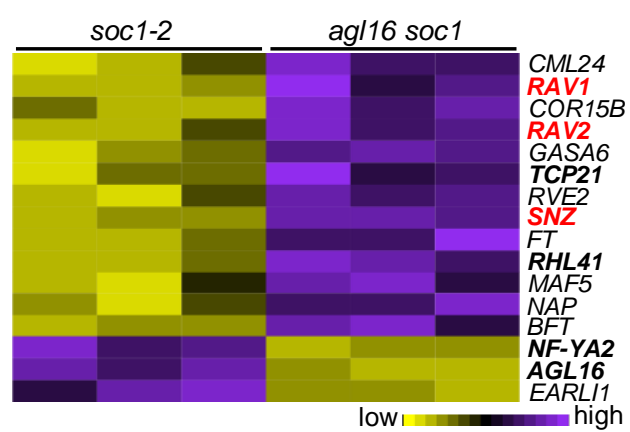

Fig. 7 The AGL16-SOC1 module collaborates on regulation of genome-wide gene expression.

A. The number of differentially expressed genes (DEGs) in three mutants. The exact number of up (red) or down (blue) regulated DEGs were given on each cone.

B and C. Heatmaps showing the normalized relative expression of soc1-2 (B) and agl16 soc1 (C) DEGs in all four lines. The boxplots in the middle gave the data distribution pattern for each cluster.

D. Venn diagram demonstrating the overlap between DEGs and the AGL16 targets profile.

E. A detailed comparison between the DEGs in soc1-2 and agl16 soc1 mutants with the AGL16 binding profile. Bold numbers in brackets showed the number of DEGs bound by AGL16.

F. A heatmap showing the normalized relative expression of the DEGs related to flowering time regulation in the soc1-2 and agl16 soc1 mutants. 

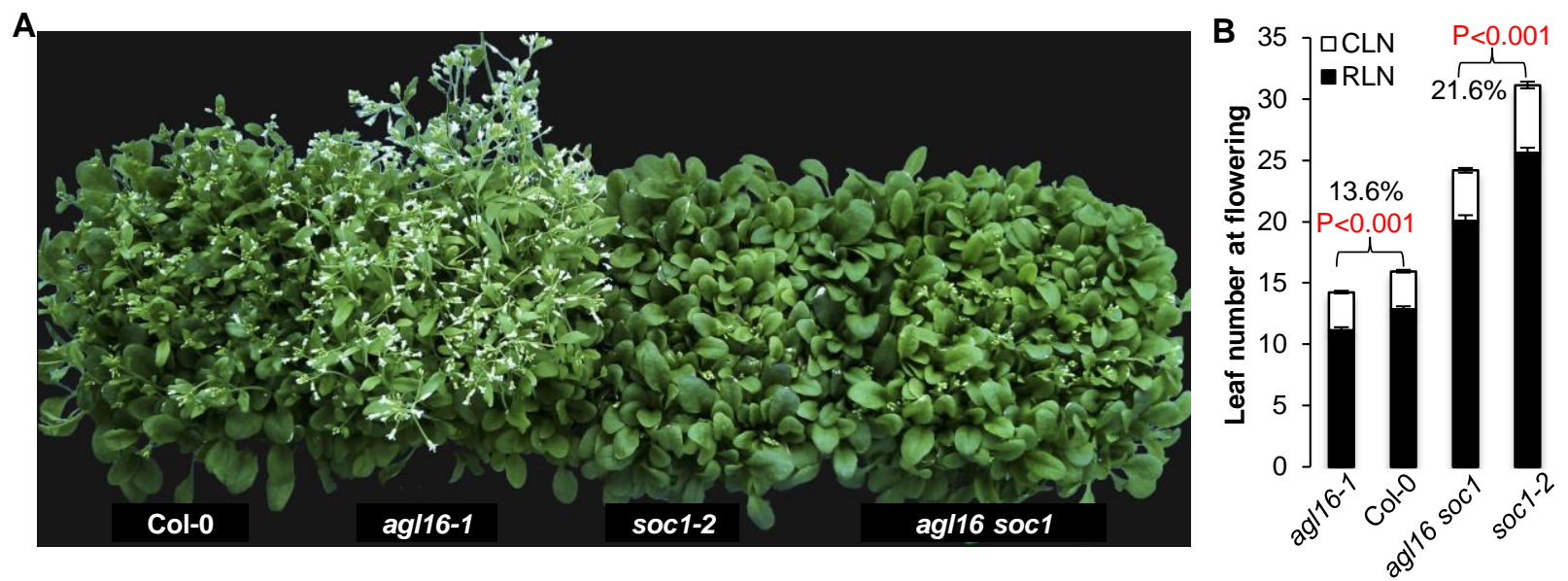

Fig. 8 AGL16 and SOC1 regulate additively flowering time.

A. Flowering behaviors of LD-growing wild type Col-0, ag/16-1, soc1-2 and ag/16 soc1 mutants.

B. Leaf number production upon flowering under LD conditions. Rosette (filled bars, RLN) and cauline (open bars, CLN) leaves were shown. Numbers in percentage showed the earlier flowering level of agl16-1 and agl16 soc 1 comparing to Col-0 and soc1-2, respectively. Analyses were repeated three times and all had similar patterns. 

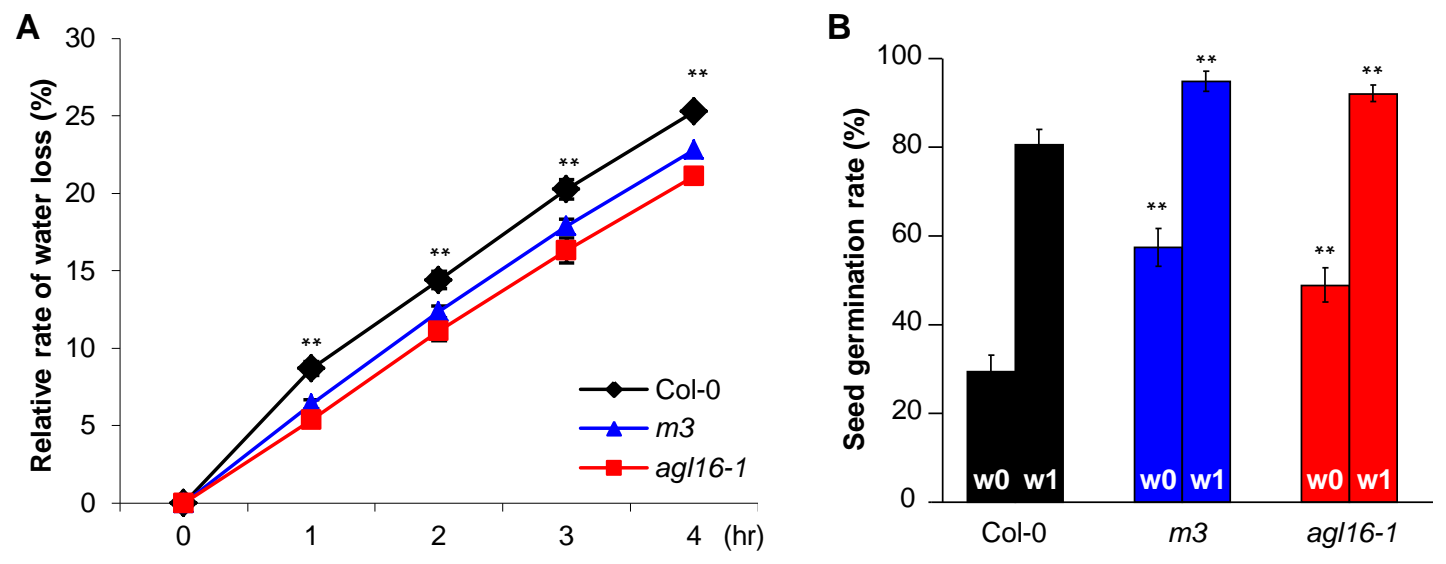

Fig. 9 miR824-AGL16 regulates water loss rate and seed dormancy.

A. Relative rates of water loss for ag/16-1 and $m 3$. Six weeks old rosettes growing under SD conditions were cut and the decreases of fresh weight in percentage were measured.

B. Changes in seed germination behavior of ag/16-1 and $m 3$ lines in contrast to Col-0. Bars mark the germination proportion in percentage at the time point of freshly harvesting (w0) and one week after (w1). In $\mathbf{A}$ and $\mathbf{B}$, mean values for at least ten individuals with standard deviation were shown. The experiments were replicated for at least twice with similar patterns. Significance was tested against Col0 with Student's $t$-test, ${ }^{* *} p<0.01$. 


\section{Parsed Citations}

Aerts, Niels, Suzanne de Bruijn, Hilda van Mourik, Gerco C. Angenent, and Aalt D. J. van Dijk. 2018. 'Comparative analysis of binding patterns of MADS-domain proteins in Arabidopsis thaliana', BMC Plant Biology, 18: 131.

Google Scholar: Author Only Title Only Author and Title

Avarez-Buylla, E. R., S. J. Liljegren, S. Pelaz, S. E. Gold, C. Burgeff, G. S. Ditta, F. Vergara-Silva, and M. F. Yanofsky. 2000. 'MADS-box gene evolution beyond flowers: expression in pollen, endosperm, guard cells, roots and trichomes', Plant J, $24: 457-66$.

Google Scholar: Author Only Title Only Author and Title

Andres, Fernando, and George Coupland. 2012. 'The genetic basis of flowering responses to seasonal cues', Nat Rev Genet, 13: 62739.

Google Scholar: Author Only Title Only Author and Title

Aukerman, M. J., and H. Sakai. 2003. 'Regulation of flowering time and floral organ identity by a MicroRNA and its APETALA2-like target genes', Plant Cell, 15: 2730-41.

Google Scholar: Author Only Title Only Author and Title

Bao, Shengjie, Changmei Hua, Gengqing Huang, Peng Cheng, Ximing Gong, Lisha Shen, and Hao Yu. 2019. 'Molecular Basis of Natural Variation in Photoperiodic Flowering Responses', Developmental Cell, 50: 90-101.e3.

Google Scholar: Author Only Title Only Author and Title

Bentsink, Leónie, and Maarten Koornneef. 2008. 'Seed Dormancy and Germination', The Arabidopsis Book: e0119.

Google Scholar: Author Only Title Only Author and Title

Bewley, J. D. 1997. 'Seed Germination and Dormancy', Plant Cell, 9: 1055-66.

Google Scholar: Author Only Title Only Author and Title

Bouche, Frederic, Guillaume Lobet, Pierre Tocquin, and Claire Perilleux. 2016. 'FLOR-ID: an interactive database of flowering-time gene networks in Arabidopsis thaliana', Nucleic Acids Research, 44: D1167-D71.

Google Scholar: Author Only Title Only Author and Title

Boxall, Susanna F., Jonathan M. Foster, Hans J. Bohnert, John C. Cushman, Hugh G. Nimmo, and James Hartwell. 2005. 'Conservation and Divergence of Circadian Clock Operation in a Stress-Inducible Crassulacean Acid Metabolism Species Reveals Clock Compensation against Stress', Plant physiology, 137: 969-82.

Google Scholar: Author Only Title Only Author and Title

Castillejo, C., and S. Pelaz. 2008. 'The balance between CONSTANS and TEMPRANILLO activities determines FT expression to trigger flowering', Curr Biol, 18: 1338-43.

Google Scholar: Author Only Title Only Author and Title

Das, S. S., S. Yadav, A Singh, V. Gautam, A K. Sarkar, A K. Nandi, P. Karmakar, M. Majee, and N. Sanan-Mishra. 2018. 'Expression dynamics of miRNAs and their targets in seed germination conditions reveals miRNA-ta-siRNA crosstalk as regulator of seed germination', Sci Rep, 8: 13.

Google Scholar: Author Only Title Only Author and Title

de Folter, S., R. G. Immink, M. Kieffer, L. Parenicova, S. R. Henz, D. Weigel, M. Busscher, M. Kooiker, L. Colombo, M. M. Kater, B. Davies, and G. C. Angenent. 2005. 'Comprehensive interaction map of the Arabidopsis MADS Box transcription factors', Plant Cell, 17: 1424-33.

Google Scholar: Author Only Title Only Author and Title

de Meaux, J., J. Y. Hu, U. Tartler, and U. Goebel. 2008. 'Structurally different alleles of the ath-MIR824 microRNA precursor are maintained at high frequency in Arabidopsis thaliana', Proc Natl Acad Sci U S A, 105: 8994-99.

Google Scholar: Author Only Title Only Author and Title

Deng, Weiwei, Hua Ying, Chris A Helliwell, Jennifer M. Taylor, W. James Peacock, and Elizabeth S. Dennis. 2011. 'FLOWERING LOCUS C (FLC) regulates development pathways throughout the life cycle of Arabidopsis', Proc Natl Acad Sci U S A, 108: 6680-85.

Google Scholar: Author Only Title Only Author and Title

Fahlgren, N., M. D. Howell, K. D. Kasschau, E. J. Chapman, C. M. Sullivan, J. S. Cumbie, S. A Givan, T. F. Law, S. R. Grant, J. L. Dangl, and J. C. Carrington. 2007. 'High-throughput sequencing of Arabidopsis microRNAs: evidence for frequent birth and death of MIRNA genes', PLoS One, 2: e219.

Google Scholar: Author Only Title Only Author and Title

Fornara, F., A de Montaigu, and G. Coupland. 2010. 'SnapShot: Control of flowering in Arabidopsis', Cell, 141: 550,50 e1-2.

Google Scholar: Author Only Title Only Author and Title

Gregis, Veronica, Fernando Andres, Alice Sessa, Rosalinda Guerra, Sara Simonini, Julieta Mateos, Stefano Torti, Federico Zambelli, Gian Prazzoli, Katrine Bjerkan, Paul Grini, Giulio Pavesi, Lucia Colombo, George Coupland, and Martin Kater. 2013. 'Identification of pathways directly regulated by SHORT VEGETATIVE PHASE during vegetative and reproductive development in Arabidopsis', Genom Biol, 14: R56.

Google Scholar: Author Only Title Only Author and Title 
bioRxiv preprint doi: https://doi.org/10.1101/2021.05.10.443448; this version posted May 10, 2021. The copyright holder for this preprint (which was not certified by peer review) is the author/funder, who has granted bioRxiv a license to display the preprint in perpetuity. It is made

Heinz, S., C. Benner, N. Spann, E. Bertolino, Y. C. Lin, P. Laslo, J. X. Cheng, C. Murre, H. Singh, and C. K. Glass. 2010. 'Simple Combinations of Lineage-Determining Transcription Factors Prime cis-Regulatory Elements Required for Macrophage and B Cell Identities', Molecular cell, 38: 576-89.

Google Scholar: Author Only Title Only Author and Title

Hu, J. Y., Y. Zhou, F. He, X. Dong, L. Y. Liu, G. Coupland, F. Turck, and J. de Meaux. 2014. 'miR824-Regulated AGAMOUS-LIKE16 Contributes to Flowering Time Repression in Arabidopsis', Plant Cell, 26: 2024-37.

Google Scholar: Author Only Title Only Author and Title

Hwang, Keumbi, Hendry Susila, Zeeshan Nasim, Ji-Yul Jung, and Ji Hoon Ahn. 2019. 'Arabidopsis ABF3 and ABF4 Transcription Factors Act with the NF-YC Complex to Regulate SOC1 Expression and Mediate Drought-Accelerated Flowering', Molecular Plant, 12 : $489-505$.

Google Scholar: Author Only Title Only Author and Title

Hyun, Y., R. Richter, C. Vincent, R. Martinez-Gallegos, A Porri, and G. Coupland. 2016. 'Multi-layered Regulation of SPL15 and Cooperation with SOC1 Integrate Endogenous Flowering Pathways at the Arabidopsis Shoot Meristem', Developmental Cell, 37: 25466.

Google Scholar: Author Only Title Only Author and Title

Immink, R. G., I. A Tonaco, S. de Folter, A Shchennikova, A D. van Dijk, J. Busscher-Lange, J. W. Borst, and G. C. Angenent. 2009. 'SEPALLATA3: the 'glue' for MADS box transcription factor complex formation', Genome Biol, 10 : R24.

Google Scholar: Author Only Title Only Author and Title

Immink, Richard G.H., David Posé, Silvia Ferrario, Felix Ott, Kerstin Kaufmann, Felipe Leal Valentim, Stefan de Folter, Froukje van der Wal, Aalt D.J. van Dijk, Markus Schmid, and Gerco C. Angenent. 2012. 'Characterization of SOC1's Central Role in Flowering by the Identification of Its Upstream and Downstream Regulators', Plant physiology, 160: 433-49.

Google Scholar: Author Only Title Only Author and Title

Johanson, Urban, Joanne West, Clare Lister, Scott Michaels, Richard Amasino, and Caroline Dean. 2000. 'Molecular Analysis of FRIGIDA, a Major Determinant of Natural Variation in Arabidopsis Flowering Time', Science, 290: $344-47$.

Google Scholar: Author Only Title Only Author and Title

Jung, J. H., Y. Ju, P. J. Seo, J. H. Lee, and C. M. Park. 2012. 'The SOC1-SPL module integrates photoperiod and gibberellic acid signals to control flowering time in Arabidopsis', Plant J, 69: 577-88.

Google Scholar: Author Only Title Only Author and Title

Jung, J. H., Y. H. Seo, P. J. Seo, J. L. Reyes, J. Yun, N. H. Chua, and C. M. Park. 2007. 'The GIGANTEAregulated microRNA172 mediates photoperiodic flowering independent of CONSTANS in Arabidopsis', Plant Cell, 19: 2736-48.

Google Scholar: Author Only Title Only Author and Title

Kaufmann, K., J. M. Muino, R. Jauregui, C. A Airoldi, C. Smaczniak, P. Krajewski, and G. C. Angenent. 2009. 'Target genes of the MADS transcription factor SEPALLATA3: integration of developmental and hormonal pathways in the Arabidopsis flower', PLoS Biol, 7: e1000090.

Google Scholar: Author Only Title Only Author and Title

Kaufmann, K., F. Wellmer, J. M. Muino, T. Ferrier, S. E. Wuest, V. Kumar, A Serrano-Mislata, F. Madueno, P. Krajewski, E. M.

Meyerowitz, G. C. Angenent, and J. L. Riechmann. 2010. 'Orchestration of floral initiation by APETALA1', Science, 328: 85-9.

Google Scholar: Author Only Title Only Author and Title

Kazan, Kemal, and John M. Manners. 2013. 'MYC2: The Master in Action', Molecular Plant, 6: 686-703.

Google Scholar: Author Only Title Only Author and Title

Kim, D., J. M. Paggi, C. Park, C. Bennett, and S. L. Salzberg. 2019. 'Graph-based genome alignment and genotyping with HISAT2 and HISAT-genotype', Nature Biotechnology, 37: 907-+.

Google Scholar: Author Only Title Only Author and Title

Kimura, Yuriko, Saya Aoki, Eigo Ando, Ayaka Kitatsuji, Aiko Watanabe, Masato Ohnishi, Koji Takahashi, Shin-ichiro Inoue, Norihito Nakamichi, Yosuke Tamada, and Toshinori Kinoshita. 2015. 'Aflowering integrator, SOC1, affects stomatal opening in Arabidopsis thaliana', Plant and Cell Physiology, 56: 640-49.

Google Scholar: Author Only Title Only Author and Title

Koornneef, M., L. Bentsink, and H. Hilhorst. 2002. 'Seed dormancy and germination', Curr Opin Plant Biol, 5: 33-6.

Google Scholar: Author Only Title Only Author and Title

Kutter, C., H. Schob, M. Stadler, F. Meins, Jr., and A Si-Ammour. 2007. 'MicroRNA-mediated regulation of stomatal development in Arabidopsis', Plant Cell, 19: 2417-29.

Google Scholar: Author Only Title Only Author and Title

Lee, J., and I. Lee. 2010. 'Regulation and function of SOC1, a flowering pathway integrator', Journal of Experimental Botany, 61: 224754.

Google Scholar: Author Only Title Only Author and Title

Lee, J., M. Oh, H. Park, and I. Lee. 2008. 'SOC1 translocated to the nucleus by interaction with AGL24 directly regulates leafy', Plant J, 55: 832-43. 
bioRxiv preprint doi: https://doi.org/10.1101/2021.05.10.443448; this version posted May 10, 2021. The copyright holder for this preprint (which was not certified by peer review) is the author/funder, who has granted bioRxiv a license to display the preprint in perpetuity. It is made

Google Scholar: Author Only Title Only Author and Title available under aCC-BY-NC-ND 4.0 International license.

Lefebvre, Valérie, Helen North, Anne Frey, Bruno Sotta, Mitsunori Seo, Masanori Okamoto, Eiji Nambara, and Annie Marion-Poll. 2006. 'Functional analysis of Arabidopsis NCED6 and NCED9 genes indicates that ABA synthesized in the endosperm is involved in the induction of seed dormancy', The Plant Journal, 45: 309-19.

Google Scholar: Author Only Title Only Author and Title

Li, D., C. Liu, L. Shen, Y. Wu, H. Chen, M. Robertson, C. A Helliwell, T. Ito, E. Meyerowitz, and H. Yu. 2008. 'A repressor complex governs the integration of flowering signals in Arabidopsis', Dev Cell, 15: 110-20.

Google Scholar: Author Only Title Only Author and Title

Li, H. 2013. 'Aligning sequence reads, clone sequences and assembly contigs with BWA-MEM', arXiv: 1303.3997.

Google Scholar: Author Only Title Only Author and Title

Li, H., B. Handsaker, A Wysoker, T. Fennell, J. Ruan, N. Homer, G. Marth, G. Abecasis, R. Durbin, and Proc Genome Project Data. 2009. 'The Sequence Alignment/Map format and SAMtools', Bioinformatics, 25: 2078-79.

Google Scholar: Author Only Title Only Author and Title

Li, Hui, Keyi Ye, Yiting Shi, Jinkui Cheng, Xiaoyan Zhang, and Shuhua Yang. 2017. 'BZR1 Positively Regulates Freezing Tolerance via CBF-Dependent and CBF-Independent Pathways in Arabidopsis', Molecular Plant, 10: 545-59.

Google Scholar: Author Only Title Only Author and Title

Liao, Y., G. K. Smyth, and W. Shi. 2014. 'featureCounts: an efficient general purpose program for assigning sequence reads to genomic features', Bioinformatics, 30: 923-30.

Google Scholar: Author Only Title Only Author and Title

Liao, Yang, Gordon K. Smyth, and Wei Shi. 2013. 'The Subread aligner: fast, accurate and scalable read mapping by seed-and-vote', Nucleic Acids Research, 41.

Liu, Tongkun, Ying Li, Jun Ren, Yu Qian, Xuedong Yang, Weike Duan, and Xilin Hou. 2013. 'Nitrate or NaCl regulates floral induction in Arabidopsis thaliana', Biologia, 68: 215-22.

Google Scholar: Author Only Title Only Author and Title

Machanick, P., and T. L. Bailey. 2011. 'MEME-ChIP: motif analysis of large DNA datasets', Bioinformatics, $27: 1696-97$.

Google Scholar: Author Only Title Only Author and Title

Mateos, Julieta L., Vicky Tilmes, Pedro Madrigal, Edouard Severing, René Richter, Colin W. M. Rijkenberg, Paweł Krajewski, and George Coupland. 2017. 'Divergence of regulatory networks governed by the orthologous transcription factors FLC and PEP1 in Brassicaceae species', Proceedings of the National Academy of Sciences, 114: E11037-E46.

Google Scholar: Author Only Title Only Author and Title

Mateos, Julieta, Pedro Madrigal, Kenichi Tsuda, Vimal Rawat, Rene Richter, Maida Romera-Branchat, Fabio Fornara, Korbinian Schneeberger, Pawe Krajewski, and George Coupland. 2015. 'Combinatorial activities of SHORT VEGETATIVE PHASE and

FLOWERING LOCUS C define distinct modes of flowering regulation in Arabidopsis', Genome Biology, $16: 31$.

Google Scholar: Author Only Title Only Author and Title

Mathieu, J., L. J. Yant, F. Murdter, F. Kuttner, and M. Schmid. 2009. 'Repression of flowering by the miR172 target SMZ, PLoS Biol, 7: e1000148.

Google Scholar: Author Only Title Only Author and Title

Mi, H. Y., X. S. Huang, A Muruganujan, H. M. Tang, C. Mills, D. Kang, and P. D. Thomas. 2017. 'PANTHER version 11: expanded annotation data from Gene Ontology and Reactome pathways, and data analysis tool enhancements', Nucleic Acids Research, 45: D183D89.

Google Scholar: Author Only Title Only Author and Title

Michaels, S. D. 2009. 'Flowering time regulation produces much fruit', Curr Opin Plant Biol, 12: 75-80.

Google Scholar: Author Only Title Only Author and Title

Michaels, S. D., and R. M. Amasino. 2001. 'Loss of FLOWERING LOCUS C activity eliminates the late-flowering phenotype of FRIGIDA and autonomous pathway mutations but not responsiveness to vernalization', Plant Cell, 13: 935-41.

Google Scholar: Author Only Title Only Author and Title

Nee, G., Y. Xiang, and W. J. J. Soppe. 2017. 'The release of dormancy, a wake-up call for seeds to germinate', Current Opinion in Plant Biology, 35: 8-14.

Google Scholar: Author Only Title Only Author and Title

Olas, Justyna Jadwiga, Judith Van Dingenen, Christin Abel, Magdalena Anna Dzialo, Regina Feil, Anne Krapp, Armin Schlereth, and Vanessa Wahl. 2019. 'Nitrate acts at the Arabidopsis thaliana shoot apical meristem to regulate flowering time', The New phytologist, 223: 814-27.

Google Scholar: Author Only Title Only Author and Title

Pajoro, Alice, Pedro Madrigal, Jose M. Muiño, José Tomás Matus, Jian Jin, Martin A Mecchia, Juan M. Debernardi, Javier F. Palatnik, Salma Balazadeh, Muhammad Arif, Diarmuid S. Ó'Maoiléidigh, Frank Wellmer, Pawel Krajewski, José-Luis Riechmann, Gerco C.

Angenent, and Kerstin Kaufmann. 2014. 'Dynamics of chromatin accessibility and gene regulation by MADS-domain transcription 
bioRxiv preprint doi: https://doi.org/10.1101/2021.05.10.443448; this version posted May 10, 2021. The copyright holder for this preprint (which was not certified by peer review) is the author/funder, who has granted bioRxiv a license to display the preprint in perpetuity. It is made

factors in flower development', Genome Biology, 15: R41. available under aCC-BY-NC-ND 4.0 International license.

Google Scholar: Author Only Title Only Author and Title

Rajagopalan, R., H. Vaucheret, J. Trejo, and D. P. Bartel. 2006. 'Adiverse and evolutionarily fluid set of microRNAs in Arabidopsis thaliana', Genes Dev, 20: 3407-25.

Google Scholar: Author Only Title Only Author and Title

Reimer, J. J., and F. Turck. 2010. 'Genome-wide mapping of protein-DNA interaction by chromatin immunoprecipitation and DNA microarray hybridization (ChIP-chip). Part A: ChIP-chip molecular methods', Methods Mol Biol, 631: 139-60.

Google Scholar: Author Only Title Only Author and Title

Richter, Rene, Atsuko Kinoshita, Coral Vincent, Rafael Martinez-Gallegos, He Gao, Annabel D. van Driel, Youbong Hyun, Julieta L. Mateos, and George Coupland. 2019. 'Floral regulators FLC and SOC1 directly regulate expression of the B3-type transcription factor TARGET OF FLC AND SVP 1 at the Arabidopsis shoot apex via antagonistic chromatin modifications', PLoS genetics, $15:$ e1008065. Google Scholar: Author Only Title Only Author and Title

Searle, I., Y. He, F. Turck, C. Vincent, F. Fornara, S. Krober, R. A Amasino, and G. Coupland. 2006. 'The transcription factor FLC confers a flowering response to vernalization by repressing meristem competence and systemic signaling in Arabidopsis', Genes Dev, 20: 898-912.

Google Scholar: Author Only Title Only Author and Title

Tao, Z, L. S. Shen, C. Liu, L. Liu, Y. Y. Yan, and H. Yu. 2012. 'Genome-wide identification of SOC1 and SVP targets during the floral transition in Arabidopsis', Plant Journal, 70: 549-61.

Google Scholar: Author Only Title Only Author and Title

Thorvaldsdottir, H., J. T. Robinson, and J. P. Mesirov. 2013. 'Integrative Genomics Viewer (IGV): high-performance genomics data visualization and exploration', Briefings in Bioinformatics, 14: 178-92.

Google Scholar: Author Only Title Only Author and Title

Tian, T., Y. Liu, H. Y. Yan, Q. You, X. Yi, Z Du, W. Y. Xu, and Z Su. 2017. 'agriGo v2.0: a GO analysis toolkit for the agricultural community, 2017 update', Nucleic Acids Research, 45: W122-W29.

Google Scholar: Author Only Title Only Author and Title

Torti, Stefano, Fabio Fornara, Coral Vincent, Fernando Andrés, Karl Nordström, Ulrike Göbel, Daniela Knoll, Heiko Schoof, and George Coupland. 2012. 'Analysis of the Arabidopsis Shoot Meristem Transcriptome during Floral Transition Identifies Distinct Regulatory

Patterns and a Leucine-Rich Repeat Protein That Promotes Flowering', Plant Cell, 24: 444-62.

Google Scholar: Author Only Title Only Author and Title

Wang, Houping, Yang Li, Jinjing Pan, Dengji Lou, Yanru Hu, and Diqiu Yu. 2017. 'The bHLH Transcription Factors MYC2, MYC3, and MYC4 Are Required for Jasmonate-Mediated Inhibition of Flowering in Arabidopsis', Molecular Plant, 10: $1461-64$.

Google Scholar: Author Only Title Only Author and Title

Xiang, Y., K. Nakabayashi, J. Ding, F. He, L. Bentsink, and W. J. J. Soppe. 2014. 'REDUCED DORMANCY5 Encodes a Protein Phosphatase 2C That Is Required for Seed Dormancy in Arabidopsis', Plant Cell, 26: 4362-75.

Google Scholar: Author Only Title Only Author and Title

Xiao, C. W., F. L. Chen, X. H. Yu, C. T. Lin, and Y. F. Fu. 2009. 'Over-expression of an AT-hook gene, AHL22, delays flowering and inhibits the elongation of the hypocotyl in Arabidopsis thaliana', Plant Molecular Biology, 71: 39-50.

Google Scholar: Author Only Title Only Author and Title

Yan, Fei-Hong, Li-Ping Zhang, Fang Cheng, Dong-Mei Yu, and Jin-Yong Hu. 2021. 'Accession-specific flowering time variation in response to nitrate fluctuation in Arabidopsis thaliana', Plant Diversity, 43: 78-85.

Google Scholar: Author Only Title Only Author and Title

Yu, G. C., L. G. Wang, and Q. Y. He. 2015. 'ChIPseeker: an R/Bioconductor package for ChIP peak annotation, comparison and visualization', Bioinformatics, 31: 2382-83.

Google Scholar: Author Only Title Only Author and Title

Zhai, Qingzhe, Xin Zhang, Fangming Wu, Hailong Feng, Lei Deng, Li Xu, Min Zhang, Qiaomei Wang, and Chuanyou Li. 2015.

'Transcriptional Mechanism of Jasmonate Receptor COI1-Mediated Delay of Flowering Time in Arabidopsis', The Plant Cell, 27: 281428.

Google Scholar: Author Only Title Only Author and Title

Zhang, Y., T. Liu, C. A Meyer, J. Eeckhoute, D. S. Johnson, B. E. Bernstein, C. Nussbaum, R. M. Myers, M. Brown, W. Li, and X. S. Liu. 2008. 'Model-based Analysis of ChIP-Seq (MACS)', Genome Biology, 9: 9.

Google Scholar: Author Only Title Only Author and Title

Zhao, C., A Hanada, S. Yamaguchi, Y. Kamiya, and E. P. Beers. 2011. 'The Arabidopsis Myb genes MYR1 and MYR2 are redundant negative regulators of flowering time under decreased light intensity', Plant J, 66: 502-15.

Google Scholar: Author Only Title Only Author and Title

Zhao, Ping-Xia, Z-Qing Miao, Jing Zhang, Si-Yan Chen, Qian-Qian Liu, and Cheng-Bin Xiang. 2020. 'MADS-box factor AGL16 negatively regulates drought resistance via stomatal density and stomatal movement', Journal of Experimental Botany, 71: $6092-610$. 
bioRxiv preprint doi: https://doi.org/10.1101/2021.05.10.443448; this version posted May 10, 2021. The copyright holder for this preprint (which was not certified by peer review) is the author/funder, who has granted bioRxiv a license to display the preprint in perpetuity. It is made Google Scholar: Author Only Title Only Author and Title available under aCC-BY-NC-ND 4.0 International license.

Zhou, Yue, Benjamin Hartwig, Geo Velikkakam James, Korbinian Schneeberger, and Franziska Turck. 2016. 'Complementary Activities of TELOMERE REPEAT BINDING Proteins and Polycomb Group Complexes in Transcriptional Regulation of Target Genes', The Plant Cell, 28: 87-101.

Google Scholar: Author Only Title Only Author and Title

Supporting information 